\title{
Intrinsic mechanism for magnetothermal conductivity oscillations in spin-orbit-coupled nodal superconductors
}

\author{
W. A. Atkinson $\odot^{1, *}$ and A. P. Kampf ${ }^{2}$ \\ ${ }^{1}$ Department of Physics and Astronomy, Trent University, Peterborough, Ontario K9L 0G2, Canada \\ ${ }^{2}$ Theoretical Physics III, Center for Electronic Correlations and Magnetism, Institute of Physics, \\ University of Augsburg, 86135 Augsburg, Germany
}

(Received 3 November 2020; revised 7 March 2021; accepted 17 March 2021; published 7 April 2021)

\begin{abstract}
We describe a mechanism by which the longitudinal thermal conductivity $\kappa_{x x}$, measured in an in-plane magnetic field, oscillates as a function of field angle in layered nodal superconductors. These oscillations occur when the spin-orbit splitting at the nodes is larger than the nodal scattering rate, and are complementary to vortexinduced oscillations identified previously. In sufficiently anisotropic materials, the spin-orbit mechanism may be dominant. As a particular application, we focus on the cuprate high-temperature superconductor $\mathrm{YBa}_{2} \mathrm{Cu}_{3} \mathrm{O}_{6+x}$. This material belongs to the class of Rashba bilayers, in which individual $\mathrm{CuO}_{2}$ layers lack inversion symmetry although the crystal itself is globally centrosymmetric. We show that spin-orbit coupling endows $\kappa_{x x} / T$ with a characteristic dependence on magnetic field angle that should be easily detected experimentally, and argue that for underdoped samples the spin-orbit contribution is larger than the vortex contribution. A key advantage of the magnetothermal conductivity is that it is a bulk probe of spin-orbit physics, and therefore not sensitive to inversion breaking at surfaces.
\end{abstract}

DOI: 10.1103/PhysRevResearch.3.023023

\section{INTRODUCTION}

Nodal superconductors are characterized by an energy gap that vanishes at point or line "nodes" on the Fermi surface. Low-energy quasiparticle excitations exist in the neighborhood of the nodes, and these excitations are reflected in characteristic power laws in the temperature dependence of various thermodynamic quantities [1]. These power laws can distinguish different node types (i.e., line versus point), but contain incomplete information about the symmetry of the superconducting state. Magnetothermal conductivity measurements are particularly useful in this regard, as the magnetic field dependence contains information about the $k$ space structure of the gap nodes [1].

A typical experiment involves the measurement of the thermal conductivity tensor $\kappa_{i j}$ in a magnetic field, which is swept through polar and/or azimuthal angles. In a nodal superconductor, the longitudinal thermal conductivity, for example, $\kappa_{x x}$, will oscillate as a function of the relative orientation of the field and the gap nodes [2,3]. The oscillation pattern has a nontrivial dependence on field strength and temperature, but with appropriate modeling will reveal the symmetry of the superconducting state [3,4]. Experimentally, this technique has been used to study the gap symmetry

\footnotetext{
*billatkinson@trentu.ca

Published by the American Physical Society under the terms of the Creative Commons Attribution 4.0 International license. Further distribution of this work must maintain attribution to the author(s) and the published article's title, journal citation, and DOI.
}

for a variety of unconventional superconductors, including organic [5] and heavy Fermion materials [1,6,7], optimally doped $\mathrm{YBa}_{2} \mathrm{Cu}_{3} \mathrm{O}_{7-\delta}$ [8-10], and $\mathrm{Sr}_{2} \mathrm{RuO}_{4}$ [11].

There are two established mechanisms underlying these oscillations, both of which are associated with the vortex lattice formed by the magnetic field. At low temperatures, circulating vortex currents "Doppler shift" the quasiparticle energies by an amount $\hbar \mathbf{v}_{s}(\mathbf{r}) \cdot \mathbf{k}$, where $\mathbf{k}$ is the quasiparticle wave vector and $\mathbf{v}_{s}(\mathbf{r})$ is the superfluid velocity in the neighborhood of $\mathbf{r}[2,12]$. The Doppler shift induces a nonzero density of states (DOS) at each node that depends on the angle between the Fermi wave vector $\mathbf{k}_{F}$ at that node and $\mathbf{v}_{s}(\mathbf{r})$. The total induced DOS, and consequently the thermal conductivity, therefore changes with the orientation of the vortex lattice or, equivalently, the field angle. At high temperatures, a second mechanism takes over, namely anisotropic quasiparticle scattering by vortices becomes the dominant source of field-angle dependence [3].

In this work, we discuss a third mechanism that is especially relevant to layered superconductors in the highly anisotropic (quasi-two-dimensional) limit. In this geometry, an in-plane magnetic field generates only weak circulating vortex currents because of the small quasiparticle mobility along the interlayer direction. Under such circumstances, we show that one may still observe pronounced field-angle oscillations in the presence of spin-orbit coupling (SOC). As a particular application of this mechanism, we focus on socalled "hidden spin-orbit" superconductors.

In the past few years, a number of materials have been discovered that exhibit signatures of SOC despite being both centrosymmetric and time-reversal symmetric [13-18]. Naive considerations would suggest this is not possible: 
by Kramers' theorem, materials that satisfy both inversion and time-reversal symmetry must have degenerate energies $E_{\mathbf{k} \uparrow}$ and $E_{\mathbf{k} \downarrow}$, which seems to eliminate the possibility of $k$-space spin textures. Such spin textures, which are a hallmark of SOC, have nonetheless been observed. Key to this is that the Kramers-degenerate states are spatially separated, which leads to spin textures that are localized in space [19-21].

Rashba bilayers form a prominent subclass of hidden spinorbit materials. In these materials, the unit cell contains pairs of conducting layers; while the unit cells are centrosymmetric, the individual layers are not. Rather, the layers are "inversion pairs," meaning that they transform into one another under inversion [20,21]. The individual layers thus exhibit some combination of Rashba and Dresselhaus SOC, with the Rashba contribution typically being larger in layered materials [20]; however, global inversion symmetry guarantees that spin textures in one layer are compensated for by opposite textures in the other layer. It is thus essential that the coupling between the layers be weak, as the $k$-space spin textures will be quenched when the two layers are strongly hybridized.

Rashba bilayers have been investigated as possible topological insulators [22], as semimetals with electrically tunable Dirac cones [23], and as model systems with nontrivial superconducting [24-27] and nematic [28,29] phases. Furthermore, many high-temperature superconductors, including $\mathrm{YBa}_{2} \mathrm{Cu}_{3} \mathrm{O}_{6+x}\left(\mathrm{YBCO}_{6+x}\right)$ and $\mathrm{Bi}_{2} \mathrm{Sr}_{2} \mathrm{CaCu}_{2} \mathrm{O}_{8+x}(\mathrm{Bi} 2212)$, satisfy the structural requirements to be Rashba bilayers; however, the relevance of this fact to cuprate physics is not established and hinges on the size of the effect.

Rashba-like spin polarization patterns have been directly measured in Bi2212 via spin-polarized angle-resolved photoemission spectroscopy (ARPES) experiments [30]. While important, these observations require independent confirmation because ARPES is a surface probe and therefore sensitive to inversion symmetry breaking at surfaces. Indirect evidence for SOC has been obtained from the magnetic breakdown energy scale that one infers from many quantum oscillation experiments in $\mathrm{YBCO}_{6+x}$ [31,32]. However, it remains open whether the observed splitting is due to spin-orbit physics [33,34], or to interlayer coupling [35]. A recent microscopic model for $\mathrm{YBCO}_{6+x}$ [36] suggests that the Fermi surface is spin-split by $\sim 10-20 \mathrm{meV}$ due to a Rashba-like SOC; however, this is an upper bound as interlayer coupling may quench spin-orbit physics.

In this work, we show that the nodal structure of the $d$-wave superconducting gap allows for an elegant and straightforward observation of spin-orbit coupling through the longitudinal thermal conductivity in a transverse magnetic field. This effect will be present whether or not the superconductor is quasi-two-dimensional (quasi-2D), but must be disentangled from Doppler shift contributions if circulating vortex currents are not negligible. Importantly, this is a bulk measurement that is insensitive to inversion symmetry breaking at sample surfaces and is complementary to other recent proposals: Kaladzhyan et al. showed that the dominant Friedel oscillation wave vectors associated with impurity scattering (as measured by scanning tunneling spectroscopy) reflect the spin-splitting of the Fermi surface and can be used to obtain the spinorbit coupling constant [37], while Raines et al. discussed the practicality of spin-Hall and Edelstein effects as probes of SOC [38].

We provide a simple description of the effect in Sec. II. While the mechanism has some similarities to the Doppler shift mechanism described in Ref. [2], there is the essential difference that a finite DOS is induced by Zeeman coupling to the quasiparticles rather than by circulating currents. Thermal conductivity calculations are described in Sec. III A, although details are left to the appendices, and results of these calculations are given in Sec. III B. We address the important question of how to distinguish spin-orbit and Doppler-shift contributions to the magnetothermal conductivity in Sec. IV. In that same section, we make an estimate that suggests that magnetothermal oscillations in $\mathrm{YBa}_{2} \mathrm{Cu}_{3} \mathrm{O}_{6.5}$ are dominated by spin-orbit effects.

\section{ORIGIN OF THE DENSITY OF STATES OSCILLATIONS}

For a singlet superconductor, the BCS Hamiltonian takes the form

$$
\begin{aligned}
\hat{H}_{\mathrm{BCS}}= & \sum_{\mathbf{k}, \sigma} \epsilon_{\mathbf{k}} c_{\mathbf{k} \sigma}^{\dagger} c_{\mathbf{k} \sigma}+\frac{1}{2} \sum_{\mathbf{k}}\left[\chi_{\mathbf{k}}\left(c_{\mathbf{k} \uparrow}^{\dagger} c_{-\mathbf{k} \downarrow}^{\dagger}-c_{\mathbf{k} \downarrow}^{\dagger} c_{-\mathbf{k} \uparrow}^{\dagger}\right)\right. \\
& + \text { H.c. }]
\end{aligned}
$$

where H.c. is the hermitian conjugate, $\epsilon_{\mathbf{k}}$ is the normal-state dispersion measured relative to the chemical potential, and $\chi_{\mathbf{k}}$ is the superconducting order parameter. The pairing term is typically simplified by making the permutation $-c_{\mathbf{k} \downarrow}^{\dagger} c_{-\mathbf{k} \uparrow}^{\dagger}=$ $c_{-\mathbf{k} \uparrow}^{\dagger} c_{\mathbf{k} \downarrow}^{\dagger}$ and recognizing that $\chi_{\mathbf{k}}=\chi_{-\mathbf{k}}$. However, Eq. (1) can be extended easily to include SOC and is therefore left as-is for this discussion.

Written in this form, Eq. (1) generates four flavors of BCS quasiparticle, described by the creation operators

$$
\begin{aligned}
& \gamma_{\mathbf{k} \uparrow+}^{\dagger}=u_{\mathbf{k}} c_{\mathbf{k} \uparrow}^{\dagger}+v_{\mathbf{k}}^{*} c_{-\mathbf{k} \downarrow}, \\
& \gamma_{\mathbf{k} \uparrow-}^{\dagger}=-v_{\mathbf{k}} c_{\mathbf{k} \uparrow}^{\dagger}+u_{\mathbf{k}} c_{-\mathbf{k} \downarrow}, \\
& \gamma_{\mathbf{k} \downarrow+}^{\dagger}=u_{\mathbf{k}} c_{\mathbf{k} \downarrow}^{\dagger}-v_{\mathbf{k}}^{*} c_{-\mathbf{k} \uparrow}, \\
& \gamma_{\mathbf{k} \downarrow-}^{\dagger}=v_{\mathbf{k}} c_{\mathbf{k} \downarrow}^{\dagger}+u_{\mathbf{k}} c_{-\mathbf{k} \uparrow},
\end{aligned}
$$

where the coherence factors are

$$
u_{\mathbf{k}}=\frac{1}{\sqrt{2}} \sqrt{1+\frac{\epsilon_{\mathbf{k}}}{E_{\mathbf{k}}}}, \quad v_{\mathbf{k}}=\frac{\chi_{\mathbf{k}}}{\sqrt{2}\left|\chi_{\mathbf{k}}\right|} \sqrt{1-\frac{\epsilon_{\mathbf{k}}}{E_{\mathbf{k}}}},
$$

and $E_{\mathbf{k}}=\sqrt{\epsilon_{\mathbf{k}}^{2}+\chi_{\mathbf{k}}^{2}}$ is the usual BCS quasiparticle excitation energy. The operators $\gamma_{\mathbf{k} \sigma s}^{\dagger}$ defined by Eqs. (2)-(5) are labeled by their spin-state $\sigma$ and band index $s= \pm$; we have followed the convention that the quasiparticle spectrum has two branches, with energies $E_{\mathbf{k} \sigma \pm}= \pm E_{\mathbf{k}}$, corresponding to the quasiparticle operators $\gamma_{\mathbf{k} \sigma \pm}^{\dagger}$. The branches are independent of $\sigma$, and are thus doubly degenerate with the upper (lower) branches empty (fully occupied) at zero temperature.

These branches are pictured in Fig. 1(a) along two cuts through the Brillouin zone for the case of a nodal $d_{x^{2}-y^{2}}$ superconductor. The locations of the cuts are indicated in Fig. 1(b), which shows also the normal-state Fermi surface and gap nodes, i.e., points on the Fermi surface where $\chi_{\mathbf{k}}$ vanishes such that $E_{\mathbf{k}}=0$. Throughout this work, the dispersion $\epsilon_{\mathbf{k}}$ is obtained from fits to the low-energy angle-resolved photoemission spectrum (Table I) [39]. In Fig. 1(a), the excitation 

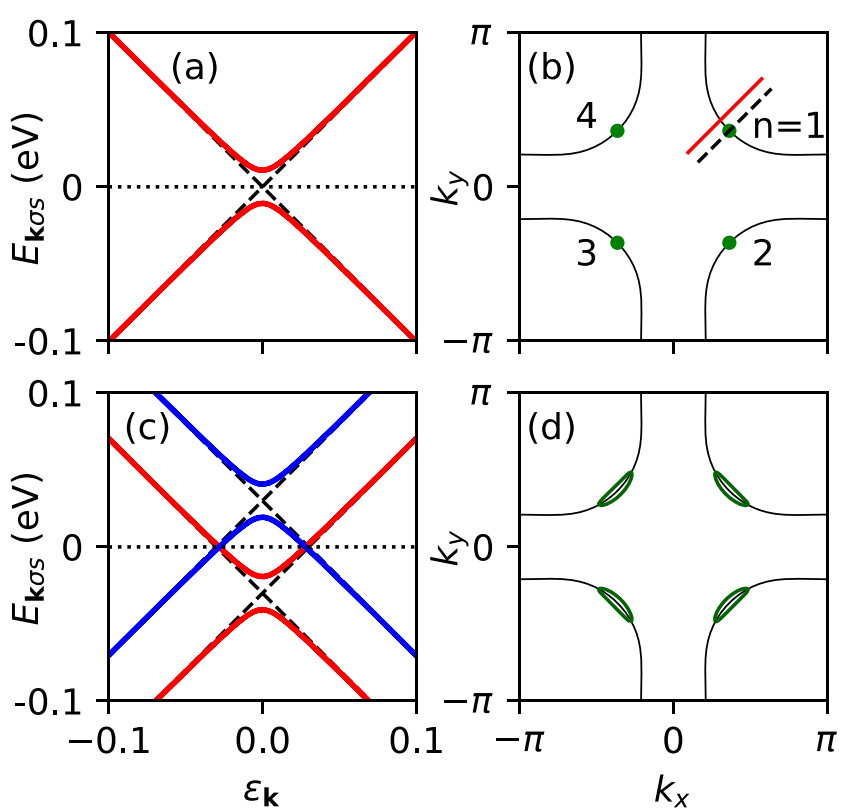
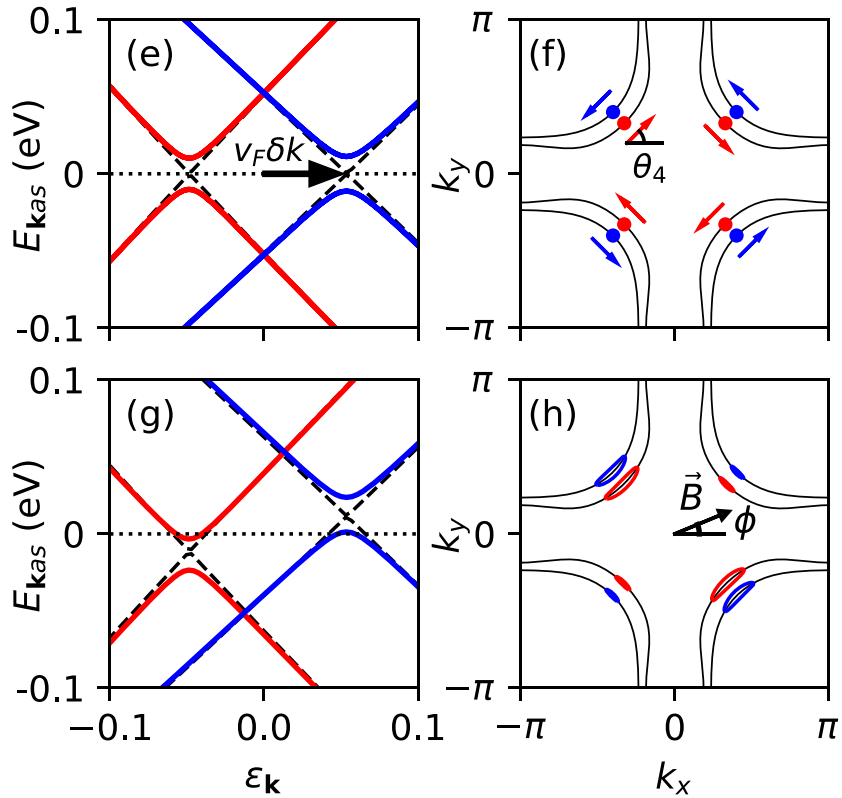

FIG. 1. Effects of a Zeeman field on quasiparticle dispersion for a 2D $d_{x^{2}-y^{2}}$ superconductor. Results are shown (a)-(d) for vanishing SOC and (e-h) for a nonzero Rashba SOC. (a) Without SOC, there are two doubly-degenerate quasiparticle branches, with energies $E_{\mathbf{k} \sigma s}=s E_{\mathbf{k}}$, with $\sigma$ the spin index and $s= \pm$ the band index. The solid (dashed) branches are plotted along the solid (dashed) cut through the Brillouin zone indicated in (b). The dispersion is gapless along cuts through any nodal point (dashed line), but is otherwise gapped (solid line). (b) For a $d_{x^{2}-y^{2}}$ superconductor, there are four nodal points (green dots) located on the normal-state Fermi surface (black line) and labeled by a nodal index $n$. (c) A magnetic Zeeman field rigidly shifts the quasiparticle branches by energies $\pm \mu B$, such that the branches are no longer degenerate. (d) These shifts inflate the nodal points to form Bogoliubov Fermi surfaces (green ellipses). (e) With SOC, bands are labeled by their helicity $a= \pm$ and band index $s= \pm$. SOC shifts the gap nodes shown in (b) by $-a \delta k$, with the red (blue) bands corresponding to $a=+$ $(a=-)$. (f) Spin-momentum locking on the normal-state Fermi surface determines the spin polarization of quasiparticle states near the gap nodes. Arrows correspond to the spin polarizations of the bands in (e). For node $n, \theta_{n}$ is defined as the smallest angle between the nodal spin polarization and the horizontal axis for the positive helicity band. (g) In an in-plane magnetic Zeeman field, the quasiparticle branches are shifted by $-a \mu B \cos \left(\theta_{n}+\phi\right)$, where $\phi$ is the angle between the field and the horizontal axis. (h) As a result, the sizes of the Bogoliubov Fermi surfaces are node-dependent. Note that the SOC $(\alpha=40 \mathrm{meV})$ and the field strength $(\mu B=30 \mathrm{meV})$ are artifically inflated for clarity. Other parameters are as described in the main text.

branches disperse linearly near the node, and are gapped away from the node.

The quasiparticles defined by Eqs. (2)-(5) have welldefined spins, such that the $z$ component of the spin operator is

$$
\begin{aligned}
\hat{S}_{z} & =\frac{1}{2} \sum_{\mathbf{k}}\left(c_{\mathbf{k} \uparrow}^{\dagger} c_{\mathbf{k} \uparrow}-c_{\mathbf{k} \downarrow}^{\dagger} c_{\mathbf{k} \downarrow}\right), \\
& =\frac{1}{4} \sum_{\mathbf{k}} \sum_{s= \pm}\left(\gamma_{\mathbf{k} \uparrow s}^{\dagger} \gamma_{\mathbf{k} \uparrow s}-\gamma_{\mathbf{k} \downarrow s}^{\dagger} \gamma_{\mathbf{k} \downarrow s}\right) .
\end{aligned}
$$

TABLE I. Tight-binding parameters for $\mathrm{YBa}_{2} \mathrm{Cu}_{3} \mathrm{O}_{6+x}$, obtained by fits to angle-resolved photoemission experiments [39]. The dispersion is $\epsilon_{\mathbf{k}}=t_{0}-2 t_{1}\left(c_{1 x}+c_{1 y}\right)+4 t_{2} c_{1 x} c_{1 y}-2 t_{3}\left(c_{2 x}+c_{2 y}\right)+$ $4 t_{4}\left(c_{2 x} c_{1 y}+c_{2 y} c_{1 x}\right)$, where $c_{n(x, y)}=\cos \left(n k_{x, y}\right)$.

\begin{tabular}{lc}
\hline \hline Parameter & Value $(\mathrm{meV})$ \\
\hline$t_{0}$ & 100 \\
$t_{1}$ & 105 \\
$t_{2}$ & 29 \\
$t_{3}$ & 25 \\
$t_{4}$ & 4 \\
\hline
\end{tabular}

A magnetic Zeeman field adds a term $-g \mu_{B} B \hat{S}_{z}$ to the electronic Hamiltonian, with $g$ the electronic $g$ factor and $\mu_{B}$ the Bohr magneton. Because $\hat{S}_{z}$ is diagonal in the quasiparticle operators $\gamma_{\mathbf{k} \sigma s}$, this additional term leaves the quasiparticles intact, but rigidly shifts their dispersions by an amount $-\mu B$ $(\sigma=\uparrow)$ or $+\mu B(\sigma=\downarrow)$, where $\mu=\frac{1}{2} g \mu_{B}$ is the electron dipole moment. These shifts are independent of the field direction (including out-of-plane fields), as the spin quantization axis is arbitrary in the absence of SOC, and is the same for each of the nodes. The resultant superconducting bands are shown in Fig. 1(c) along the same cuts as in Fig. 1(a). The band shifts inflate the nodal points to form so-called Bogoliubov Fermi surfaces [40] that separate occupied and empty quasiparticle states. These are shown as green ellipses in Fig. 1(d); they are the same for all nodes and are independent of field direction [41].

The situation changes when the SOC is nonzero. Here, the size of the induced Bogoliubov Fermi surfaces varies from node to node and depends on the field angle. For a generic 2D $d_{x^{2}-y^{2}}$ superconductor with Rashba SOC, the Hamiltonian is $\hat{H}=\sum_{\mathbf{k}}{ }^{\prime} \mathbf{C}_{\mathbf{k}}^{\dagger} \mathbf{H}_{\mathbf{k}} \mathbf{C}_{\mathbf{k}}$, with

$$
\mathbf{H}_{\mathbf{k}}=\left[\begin{array}{cc}
\mathbf{h}_{\mathbf{k}} & \Delta_{\mathbf{k}} \\
\Delta_{\mathbf{k}}^{\dagger} & -\mathbf{h}_{-\mathbf{k}}^{T}
\end{array}\right],
$$


where $\mathbf{C}_{\mathbf{k}}=\left(\begin{array}{llll}c_{\mathbf{k} \uparrow}, & c_{\mathbf{k} \downarrow}, & c_{-\mathbf{k} \uparrow}^{\dagger}, & c_{-\mathbf{k} \downarrow}^{\dagger}\end{array}\right)^{T}$, the prime indicates the summation is over a reduced Brillouin zone $\left(k_{x}, k_{y}\right) \in[0, \pi] \otimes[-\pi, \pi]$, and

$$
\begin{aligned}
\mathbf{h}_{\mathbf{k}} & =\epsilon_{\mathbf{k}} \tau_{0}+\left(\mathbf{g}_{\mathbf{k}}-\mu \mathbf{B}\right) \cdot \boldsymbol{\tau}, \\
\boldsymbol{\Delta}_{\mathbf{k}} & =i \tau_{y} \chi_{d \mathbf{k}}+i\left(\mathbf{d}_{\mathbf{k}} \cdot \boldsymbol{\tau}\right) \tau_{y}, \\
\mathbf{g}_{\mathbf{k}} & =\alpha\left(\sin k_{y},-\sin k_{x}, 0\right) .
\end{aligned}
$$

Here, $\mathbf{h}_{\mathbf{k}}$ is the Hamiltonian for the normal state, $\boldsymbol{\tau}$ and $\tau_{0}$ are the Pauli spin matrices, $\mathbf{g}_{\mathbf{k}}$ is the Rashba spin-orbit term, and $\mathbf{B}$ is the in-plane magnetic field. As shown in Fig. 1(f), SOC splits the normal-state Fermi surface into spin-polarized bands, with the electron spins locked to their momentum.

For generality, the superconducting order parameter in Eq. (10) includes both a singlet part $\chi_{d \mathbf{k}}$ and a triplet part $\mathbf{d}_{\mathbf{k}}$ [42]. The latter is formally required because a nominally singlet superconductor must develop a triplet component in response to the SOC. While mixed-parity states with comparable singlet and triplet components are possible, the more common situation is that one component is dominant [42]. Our numerical calculations for $\mathrm{YBCO}_{6+x}$, for example, find that the triplet amplitudes are of order $1 \%$ of the singlet component (see Appendix A). We thus drop the triplet component from our calculations.

For a pure $d_{x^{2}-y^{2}}$ superconductor, SOC shifts the nodes shown in Fig. 1(a) by displacements $\pm \delta k$ [Figs. 1(e) and 1(f)], where $\delta k \sim \alpha / v_{F}$. This doubles the number of nodes in each quadrant of the Brillouin zone, but the dispersion near each of the shifted nodes has the same structure as when SOC is absent [Fig. 1(e)]. (Note that although calculations are performed in a reduced Brillouin zone, we continue to show the full zone for illustrative purposes.)

In most SOC materials, the coupling constant $\alpha$ is orders of magnitude larger than $\mu B \sim 1 \mathrm{meV}$, and in this limit the physics of the nodal dispersion is easily understood. Crucially, the SOC selects a preferred polarization axis near each of the gap nodes, and this axis is largely unchanged by the Zeeman field in the limit $\mu B \ll \alpha$. In each quadrant (labeled $n=1, \ldots, 4)$ one may locally rotate the spin-quantization axis such that the Hamiltonian for each band has a BCS-like form (Appendix B). The quasiparticle creation operators are then similar to Eqs. (2)-(5) near the gap nodes, but with "up" and "down" spin directions aligned with the red and blue arrows, respectively, in Fig. 1(f). For quadrant $n$, we denote the angle between the "up" direction and the $k_{x}$ axis by $\theta_{n}$.

In the limit $\mu B \ll \alpha$, the principal effect of the Zeeman field is to shift the nodal dispersions by an amount $-a \mu B \cos \left(\theta_{n}+\phi\right)$, where $\phi$ is the angle between the magnetic field and the $k_{x}$ axis, and $a= \pm$ is the helicity of the band (positive helicity indicates that the spin winds clockwise around the center of the Brillouin zone). This has several consequences. First, the dispersions near the two spin-split nodes are shifted in opposite directions because their helicities are opposite [Fig. 1(g)]; however, the size of the induced nodal Fermi surfaces is nearly the same [Fig. 1(h)] and the spin-split nodes make nearly identical contributions to the thermal conductivity. Second, nodes belonging to different quadrants of the Brillouin zone experience different shifts reflecting the different values of $\theta_{n}$, so that the sizes of the
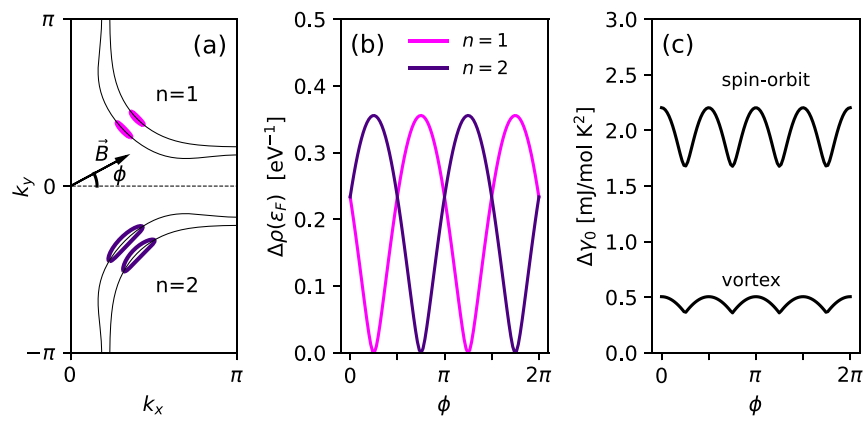

FIG. 2. Density of states induced by an in-plane Zeeman field as a function of field angle. (a) Fermi surface structure in the reduced Brillouin zone. The sizes of the Bogoliubov Fermi surfaces depend on the nodal index $n$ and on the field angle $\phi$. Fermi surfaces with $n=1$ (magenta) vanish when $\phi=\left(m+\frac{1}{4}\right) \pi$, while those with $n=2$ (violet) vanish when $\phi=\left(m-\frac{1}{4}\right) \pi$, with $m \in \mathbb{Z}$. (b) The DOS (per unit cell) induced by the Zeeman field is shown for each pair of gap nodes in (a). $\Delta \rho\left(\varepsilon_{F}\right)$ is calculated using Eq. (C4) with realistic parameters for $\mathrm{YBa}_{2} \mathrm{Cu}_{3} \mathrm{O}_{6.5}\left(a_{0}=3.8 \AA, v_{F}=1.2 \mathrm{eV} \AA\right.$, $v_{2}=0.017 \mathrm{eVA}$, and $\gamma=0.1 \mathrm{meV}$ ). (c) Corresponding oscillations of the specific heat coefficient $\Delta \gamma_{0}$ on field angle are a signature of Rashba spin-orbit coupling. For comparison, the estimated specific heat coefficients from circulating vortex currents are included (see Sec. IV). Results in (b) and (c) are for $T \rightarrow 0$, and $\mu B=1 \mathrm{meV}$, which corresponds to $B=17 \mathrm{~T}$.

induced Bogoliubov Fermi surfaces are different [Fig. 1(h)]. Finally, the relative sizes of the different nodal Fermi surfaces depend on the angle $\phi$ of the magnetic field.

The sizes of the Fermi surface pockets [Fig. 2(a)] are directly related to the induced DOS $\Delta \rho\left(\varepsilon_{F}\right)$ at the Fermi energy. Analytic expressions for $\Delta \rho\left(\varepsilon_{F}\right)$ may be obtained (Appendix C), and their field-angle-dependence, shown for each of the nodal regions in Fig. 2(b) is a signature of Rashba SOC. The two regions oscillate out of phase with each other, and the total induced DOS obtained from their sum has minima at field angles $\phi=\left(m \pm \frac{1}{4}\right) \pi$. This is reflected in the linear specific heat coefficient,

$$
\Delta \gamma_{0}=\lim _{T \rightarrow 0} \frac{\Delta c_{v}}{T}=\frac{\pi^{2}}{3} k_{B}^{2} \Delta \rho\left(\epsilon_{F}\right),
$$

shown in Fig. 2(c). The size of the oscillations depends on both $\mathbf{B}$ and on the single-particle scattering rate $\gamma$. For the benchmark case of $\mathrm{YBa}_{2} \mathrm{Cu}_{3} \mathrm{O}_{6.5}$ shown in Fig. 2(c), the induced specific heat coefficient $\Delta \gamma_{0}$ is comparable to typical measured values for an out-of-plane magnetic field (i.e. for the vortex phase) [43]. Significantly, the predicted magnetothermal oscillations are a factor of 4 larger than the expected vortex contributions for an in-plane field. In Fig. 2(c), the vortex contribution is calculated using an estimate from Ref. [2] and is discussed in detail in Sec. IV.

To obtain quantitative estimates for $\mathrm{YBa}_{2} \mathrm{Cu}_{3} \mathrm{O}_{6.5}$, we have used a dispersion $\epsilon_{\mathbf{k}}$ that was obtained from a tight-binding fit to ARPES measurements on $\mathrm{YBCO}_{6+x}$ [39], and a realistic gap function $\chi_{d \mathbf{k}}=\chi_{d}\left(\cos k_{x}-\cos k_{y}\right) / \sqrt{2}$ with $\chi_{d}=$ $50 \mathrm{meV}$. These choices give a nodal Fermi velocity $v_{F}=$ $1.2 \mathrm{eVA}$, in close agreement with experiments [44], and a superconducting nodal group velocity $v_{2}=\left|\nabla_{\mathbf{k}} \Delta_{\mathbf{k}}\right|_{\text {node }}=$ $0.17 \mathrm{eV} \AA$. Unless specified otherwise, the Rashba coupling 
constant is taken to be $\alpha=10 \mathrm{meV}$ throughout this work, which gives a spin splitting at the gap nodes of $25 \mathrm{meV}$ [36].

Although our explanation of the DOS oscillations is based on the weak-field limit, the effect is general provided the spin-splitting of the nodes is greater than the quasiparticle scattering rate $\gamma$. The effect is present regardless of the dimensionality of the system and will dominate over the vortex contribution in highly anisotropic materials; however, even in three dimensional materials the SOC and vortex effects can be comparable.

We finish this section with a comment that is specific to Rashba bilayers. The model explored in this section describes a single $\mathrm{CuO}_{2}$ layer, and there are two issues that might limit its applicability to the bilayer. First, in a Rashba bilayer, the sign of $\alpha$ is opposite in each layer, and it is a concern that the contributions from each layer might cancel. However, from Fig. 2(a), it is clear that reversing the direction of each nodal polarization will have no effect on the induced DOS. Indeed, as we show explicitly below, the field-angle dependence of the thermal conductivity is an even function of $\alpha$. Second, one must keep in mind that hybridization of the two layers via a hopping matrix element $t_{\perp}$ will quench the spin polarization. As discussed elsewhere $[33,34,36]$, the spin polarization at the gap nodes is of order $\alpha / \sqrt{t_{\perp}^{2}+\alpha^{2}}$. The analysis contained in this work assumes that $t_{\perp} \ll \alpha$, which is supported by an apparent collapse of bilayer splitting at the gap nodes in underdoped $\mathrm{YBCO}_{6+x}$ [45]. An experimental failure to measure the predicted thermal conductivity oscillations likely implies that the limit $t_{\perp} \ll \alpha$ does not apply.

\section{THERMAL CONDUCTIVITY}

\section{A. Calculations}

In this section, we discuss calculations of the longitudinal thermal conductivity in the presence of an in-plane magnetic Zeeman field. As in the previous section, we assume that the triplet contribution to the superconducting order parameter can be neglected. For $\mathrm{YBCO}_{6+x}$, we have checked numerically that neglect of the triplet components has no observable effect on the calculated longitudinal thermal conductivity. This is essentially different, then, from the intrinsic thermal Hall effect in a perpendicular Zeeman field, which depends crucially on the triplet component; together with SOC, a perpendicular Zeeman field creates a gapful mixedparity topological superconductor [46-48] whose finite Chern number determines the $T$-linear part of the thermal Hall conductivity [49].

Following Refs. [50,51], the thermal current operator is

$$
\mathbf{J}_{Q}=-\frac{i}{2} \sum_{\mathbf{k}}^{\prime} \sum_{i, j} \mathbf{V}_{\mathbf{k}, i j}\left(\dot{C}_{\mathbf{k}, i}^{\dagger} C_{\mathbf{k}, j}-C_{\mathbf{k}, i}^{\dagger} \dot{C}_{\mathbf{k}, j}\right),
$$

where $\dot{C}_{\mathbf{k}, i}$ indicates a time derivative of $C_{\mathbf{k}, i}$ and the subscripts $i$ and $j$ are elements in the four-component operator space introduced in Eq. (8). The $4 \times 4$ velocity matrix is

$$
\mathbf{V}_{\mathbf{k}}=\left[\begin{array}{cc}
\mathbf{v}_{\mathbf{k}} & \mathbf{v}_{\Delta, \mathbf{k}} \\
\mathbf{v}_{\Delta, \mathbf{k}}^{\dagger} & \mathbf{v}_{-\mathbf{k}}^{T}
\end{array}\right]
$$

In this expression, $\mathbf{v}_{\mathbf{k}}=\nabla_{\mathbf{k}} \mathbf{h}_{\mathbf{k}}$ and $\mathbf{v}_{\Delta, \mathbf{k}}=\nabla_{\mathbf{k}} \boldsymbol{\Delta}_{\mathbf{k}}$, with $\mathbf{h}_{\mathbf{k}}$ and $\boldsymbol{\Delta}_{\mathbf{k}}$ given by Eqs. (9) and (10), respectively. Equation (13) does not include corrections due to circulating thermal currents that appear when time-reversal symmetry is broken [52], as these do not contribute to the longitudinal thermal conductivity.

From the Kubo formula, the longitudinal thermal conductivity satisfies

$$
\frac{\kappa_{x x}}{T}=-\frac{\pi}{\hbar d T^{2}} \int_{-\infty}^{\infty} d x x^{2} \frac{\partial f(x)}{\partial x} \Pi^{x x}(x) .
$$

where $f(x)$ is the Fermi function, $d$ is the mean interlayer distance (for the bilayer case of $\mathrm{YBCO}_{6+x}$, it is the $c$-axis lattice constant divided by two), and where

$$
\Pi^{x x}(x)=\frac{\hbar^{2}}{2 N_{k} a_{0}^{2}} \sum_{\mathbf{k}}^{\prime} \operatorname{Tr}\left[\mathbf{A}_{\mathbf{k}}(x) \mathbf{V}_{\mathbf{k}} \mathbf{A}_{\mathbf{k}}(x) \mathbf{V}_{\mathbf{k}}\right]
$$

is the dimensionless thermal conductivity kernel. In Eq. (16), $N_{k}$ is the number of k-points in the reduced Brillouin zone, $a_{0}$ is the lattice constant, and $\mathbf{A}_{\mathbf{k}}(x)$ is the spectral function obtained from the Hamiltonian $\mathbf{H}_{\mathbf{k}}$,

$$
\mathbf{A}_{\mathbf{k}}(x)=\frac{1}{2 \pi i}\left[\left(x-i \gamma-\mathbf{H}_{\mathbf{k}}\right)^{-1}-\left(x+i \gamma-\mathbf{H}_{\mathbf{k}}\right)^{-1}\right],
$$

with $\gamma$ the quasiparticle scattering rate. At low temperature, $\kappa_{x x}$ is linear in $T$, with Eq. (15) simplifying to

$$
\frac{\kappa_{x x}}{T}=\frac{\pi^{3} k_{B}^{2}}{3 \hbar d} \Pi^{x x}(0) .
$$

In the limit of strong SOC, $|\alpha| \gg \mu B, \gamma$, it is further possible to obtain an analytic result for $\Pi^{x x}(x)$ (see Appendix B),

$$
\begin{aligned}
\Pi^{x x}(x)= & \frac{1}{8 \pi^{3}} \frac{v_{F}^{2}+v_{2}^{2}}{v_{F} v_{2}}\left\{4+\sum_{ \pm} \mathcal{E}\left[\frac{x \pm \mu B \cos \left(\frac{\pi}{4}+\phi\right)}{\gamma}\right]\right. \\
& \left.+\sum_{ \pm} \mathcal{E}\left[\frac{x \pm \mu B \cos \left(\frac{3 \pi}{4}+\phi\right)}{\gamma}\right]\right\}
\end{aligned}
$$

with $v_{2}=\left|\nabla_{\mathbf{k}} \chi_{\mathbf{k}}\right|_{\text {node }}$ and

$$
\mathcal{E}(y)=\left(y+\frac{1}{y}\right) \tan ^{-1} y .
$$

Note that, in the large- $\alpha$ limit, $\Pi^{x x}(x)$ is independent of $\alpha$.

\section{B. Results}

Figure 3 shows the longitudinal thermal conductivity in an in-plane magnetic field, as a function of the angle between the field and the $x$ axis. We focus initially on the large-SOC limit, with $\alpha=10 \mathrm{meV}$, since this is the regime that we expect to be relevant to $\mathrm{YBCO}_{6+x}$. The essential point of this figure is that at sufficiently low temperatures, $\kappa_{x x} / T$ exhibits clear and pronounced oscillations. The size of the oscillations is approximately proportional to $\mu B / \gamma$, and grows by an order of magnitude between the intermediate scattering [Figs. 3(a) and 3(b)] and clean [Fig. 3(c)] limits. The shape of the oscillations also depends on the ratio $\mu B / \gamma$, taking an approximately sinusoidal form when $\mu B \sim \gamma$, and deviating strongly from it when $\mu B \gg \gamma$. The figure also shows that the approximate 

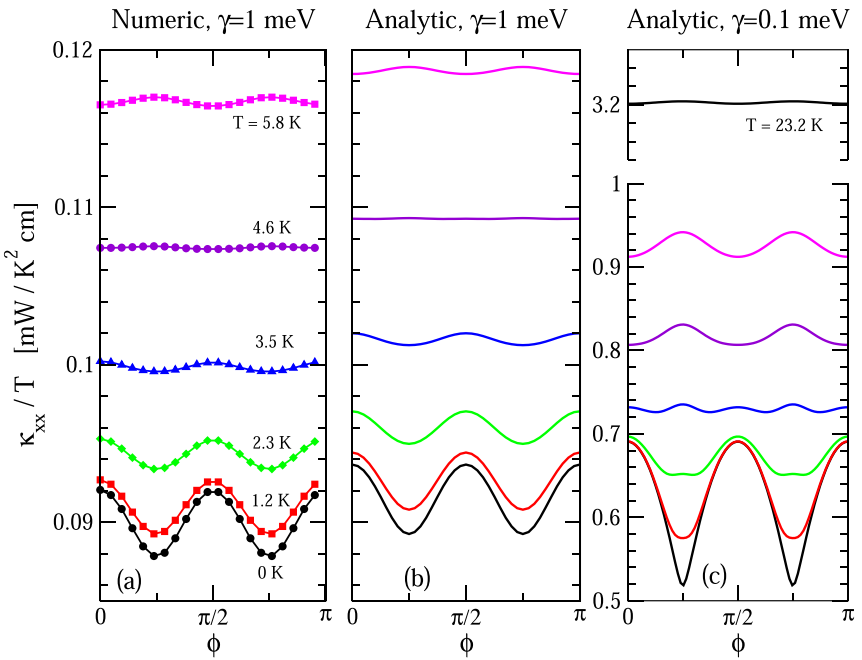

FIG. 3. Thermal conductivity in the strong SOC limit $(|\alpha| \gg$ $\mu B, \gamma)$. The thermal conductivity is shown as a function of the angle $\phi$ between the magnetic field and the $x$ axis for a range of temperatures between $T=0 \mathrm{~K}$ and $T=5.8 \mathrm{~K}=0.5 \mathrm{meV}$. Curves are generated for (a) the numerical evaluation of Eqs. (15) and (16), and (b) and (c) the analytical approximation Eq. (19). The magnetic field is $\mu B=2 \mathrm{meV}$, which (assuming $\mu=\mu_{B}$ ) corresponds to $B=34 \mathrm{~T}$. In (c), an additional curve is shown for $T=23.2 \mathrm{~K}$, corresponding to $k_{B} T=\mu B$. The SOC constant is $\alpha=10 \mathrm{meV}$. (a) and (b) are for an intermediate scattering rate, $\gamma=1 \mathrm{meV}$, and demonstrate that the analytical result is quantitatively accurate when the SOC is large; (c) is in the clean limit, $\gamma=0.1 \mathrm{meV}$, which is appropriate for $\mathrm{YBCO}_{6.5}$. Note that the scale in (c) is an order or magnitude larger than in (a) and (b).

expression (19) for the transport kernel $\Pi^{x x}(x)$ works well when $\alpha$ is large.

The oscillations shown in Fig. 3 change their qualitative character as a function of temperature. At low $T, \kappa_{x x}$ is maximal when the field angle is an integer multiple of $\frac{\pi}{2}$; above a crossover temperature $k_{B} T_{\text {cross }} \approx \mu B / 5$, the oscillations are inverted, with minima at integer multiples of $\frac{\pi}{2}$. The oscillations persist up to $k_{B} T \sim \mu B$, at which point the angle dependence can barely be discerned [Fig. 3(c), for $T=23.2 \mathrm{~K}$ ].

Because we focus on low temperatures, both the scattering rate and superconducting gap are treated as constant in our calculations. The inversion of the oscillation pattern is therefore entirely due to the thermal factor in Eq. (15), and can be traced back to the dependence of the DOS, $\rho(\phi, x)$, on field angle and energy. A simple explanation of this effect can be obtained in the clean limit. In zero field, the low-energy DOS for a $d$-wave superconductor is $\rho(x) \propto|x|$; that is, it vanishes at energy $x=0$, and rises linearly with $|x|$. In an in-plane field, the band energies are shifted by $\pm \mu B \cos \left(\theta_{n}+\phi\right)$, where the sign depends on the helicity of the band [recall Fig. $1(\mathrm{~g})$ ]. The total low-energy DOS is obtained by summing the shifted contribution from each of the bands $( \pm)$ and nodal regions $(n)$,

$$
\rho(\phi, x) \propto \sum_{ \pm} \sum_{n=1}^{2}\left|x \pm \mu B \cos \left(\theta_{n}+\phi\right)\right|,
$$

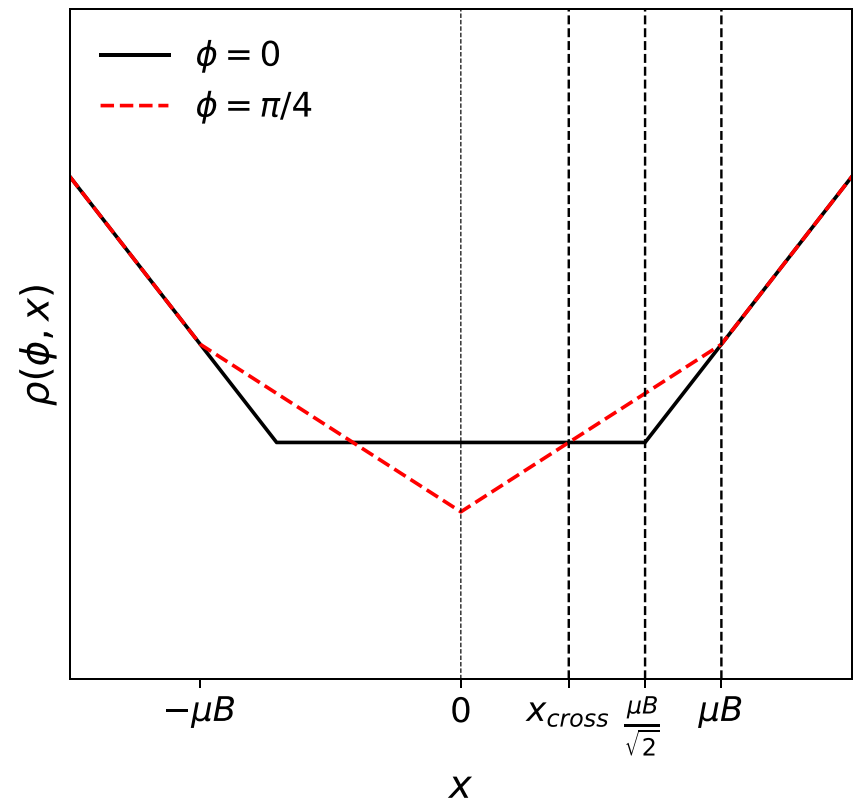

FIG. 4. Schematic densities of states for a $d$-wave superconductor in an in-plane Zeeman field at field angles $\phi=0$ and $\phi=\frac{\pi}{4}$. Plots are obtained from Eqs. (23) and (24). The densities of states cross at $x_{\text {cross }}=\mu B(\sqrt{2}-1)$.

where $\theta_{1}=\frac{\pi}{4}$ and $\theta_{2}=\frac{3 \pi}{4}$. This same term appears in Eq. (19), where for $\mu B \gg \gamma$ one may write

$$
\mathcal{E}\left[\frac{x \pm \mu B \cos \left(\theta_{n}+\phi\right)}{\gamma}\right] \approx \frac{\pi}{2 \gamma}\left|x \pm \mu B \cos \left(\theta_{n}+\phi\right)\right| \text {. }
$$

Thus, from Eq. (19), $\Pi^{x x}(x)$ in the clean limit is equal to a constant plus a term proportional to $\rho(\phi, x)$.

When $\phi=0, \cos \left(\theta_{n}+\phi\right)= \pm \frac{1}{\sqrt{2}}$, and

$$
\rho(0, x) \propto\left\{\begin{array}{ll}
4 \frac{\mu B}{\sqrt{2}}, & |x|<\frac{\mu B}{\sqrt{2}} \\
4|x|, & |x|>\frac{\mu B}{\sqrt{2}}
\end{array} .\right.
$$

Conversely, when $\phi=\frac{\pi}{4}$, the cosine is zero for $n=1$, and \pm 1 for $n=2$, yielding

$$
\rho\left(\frac{\pi}{4}, x\right) \propto\left\{\begin{array}{ll}
2|x|+2 \mu B, & |x|<\mu B \\
4|x|, & |x|>\mu B
\end{array} .\right.
$$

The two densities of states curves, shown schematically in Fig. 4 , cross at $x_{\text {cross }}=(\sqrt{2}-1) \mu B$. Because of the direct link between $\Pi^{x x}(x)$ and the DOS, $x_{\text {cross }}$ sets the temperature scale for the inversion of the thermal conductivity oscillations. The actual crossover temperature, $k_{B} T_{\text {cross }} \approx \mu B / 5$ is smaller than $x_{\text {cross }}$ by a factor of approximately 2 because of the weighting factor $x^{2} \partial f(x) / \partial x$ in Eq. (15).

A second energy scale is apparent in Fig. 4, namely, the DOS is independent of $\phi$ when $|x|>\mu B$. This sets the temperature scale at which the thermal conductivity oscillations die out.

Having understood the temperature dependence of $\kappa_{x x}$, we now examine the dependence on other model parameters. Inspection of Eq. (19) reveals that, in the zero-temperature limit, $\kappa_{x x} / T$ is a function of $\mu B / \gamma$, and is independent of $\alpha$. This is apparent in Fig. 5(a), which shows that the oscillation 

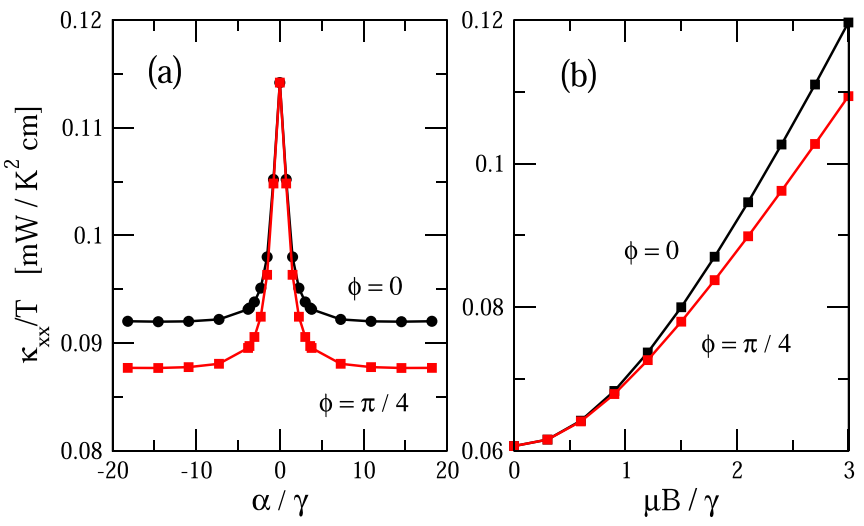

FIG. 5. Numerical results for the thermal conductivity as a function of (a) spin-orbit coupling strength and (b) magnetic field. Results are shown at field angles $\phi=0$ and $\phi=\pi / 4$; in each plot, the difference between the two curves gives the amplitude of the oscillation. Except where indicated otherwise, results are for $\alpha=10 \mathrm{meV}$, $\mu B=2 \mathrm{meV}, \gamma=1 \mathrm{meV}$, and $T=0 \mathrm{~K}$.

amplitude at $T=0$ saturates at an approximately constant value when $|\alpha| \gg \gamma$. We also note that the amplitude is a symmetric function of $\alpha$, confirming our earlier assertion that the thermal conductivity oscillations due to the two layers making up a Rashba bilayer are additive.

Figure 5(b) shows the dependence of $\kappa_{x x} / T$ on magnetic field strength. When $\mu B \rightarrow 0$, the conductivity kernel becomes independent of the scattering rate [51],

$$
\Pi^{x x}(0) \rightarrow \frac{1}{\pi^{3}} \frac{v_{F}^{2}+v_{2}^{2}}{v_{F} v_{2}},
$$

yielding $\lim _{T \rightarrow 0} \kappa_{x x} / T=0.062 \mathrm{~mW} \mathrm{~K}^{-2} \mathrm{~cm}^{-1}$. This value is close to that measured by Sutherland et al. in $\mathrm{YBCO}_{6.54}$ [53]. When $\mu B$ is not zero, the field dependence reflects the structure of the function $\mathcal{E}(\mu B / \gamma)$ in Eq. (19). For small argument, the field dependence is quadratic, with $\mathcal{E}(\mu B / \gamma) \approx$ $1+\frac{2}{3}(\mu B / \gamma)^{2}$, while for large arguments it is linear with $\mathcal{E}(\mu B / \gamma) \approx \pi \mu B / 2 \gamma$.

The value of $\gamma$ is thus central to the observability of the thermal conductivity oscillations, which are suppressed when $\gamma>\mu B$. Angle-resolved photoemission spectroscopy (ARPES) experiments have placed an upper bound of $\gamma=$ $12 \mathrm{meV}$ on the nodal scattering rate for the bilayer cuprate superconductor Bi2212 [54], consistent with the strong inhomogeneity observed in that material in tunneling experiments [55,56]. Spin-orbit effects might thus be hard to observe in Bi2212. Conversely, microwave conductivity measurements have found very small transport scattering rates $\gamma_{\text {tr }} \lesssim 0.1 \mathrm{meV}$ in $\mathrm{YBCO}_{6.50}$ at low temperatures [57]. The reasonable assumption $\gamma_{\mathrm{tr}} \sim \gamma$ places $\mathrm{YBCO}_{6.50}$ in the clean limit, where thermal conductivity oscillations should be easily observable.

Figure 6 shows the range of behavior that can be expected for $\kappa_{x x} / T$ in the clean limit for different values of $\alpha$ spanning $|\alpha| \ll \mu B$ to $|\alpha| \gg \mu B$. For comparison, the analytic result for large SOC is also plotted, and is quantitatively similar to the numerical data for all values of $\alpha$. Remarkably, there is very little to distinguish the different thermal conductivity
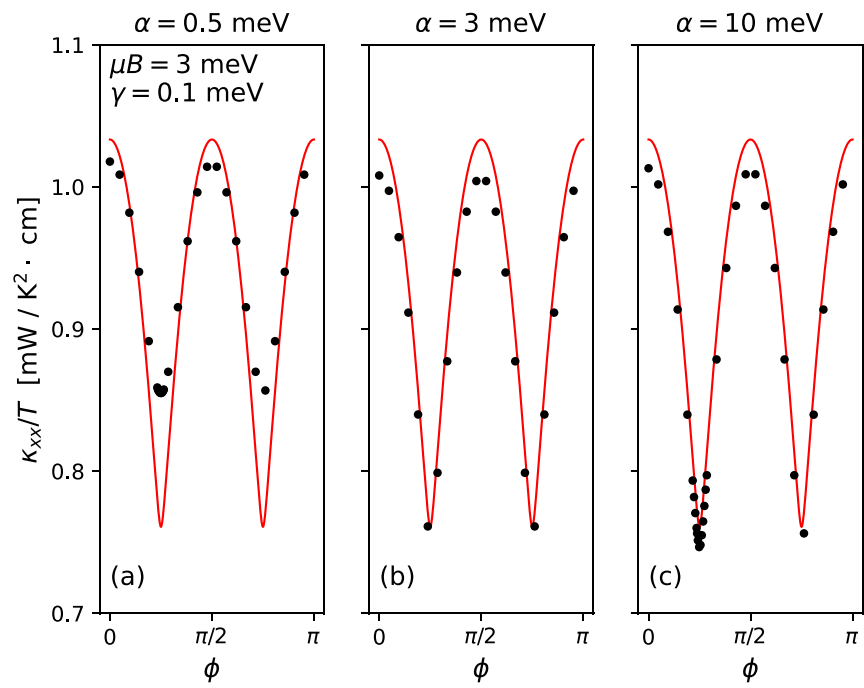

FIG. 6. Thermal conductivity in the clean limit, $\gamma \ll|\alpha|, \mu B$, as a function of field angle. Numerical data (circles) are compared to the analytical result obtained from, Eqs. (18) and (19). Equation (19) is nominally valid for $|\alpha| \gg \mu B$ but provides a good quantitative fit to the data even for $\alpha=\mu B$. Results are for $T=0, \mu B=3 \mathrm{meV}$, and $\gamma=0.1 \mathrm{meV}$.

data sets, even though $\alpha$ changes by a factor of 20 across the figure. Thus pronounced oscillations in the thermal conductivity should be observable so long as $|\alpha|>\gamma$. By fitting Eq. (19) to experimental measurements of $\kappa_{x x} / T$ at low $T$ and for $\mu B \lesssim|\alpha|$, it is possible to extract both the ratio $v_{F} / v_{2}$ and the scattering rate $\gamma$.

\section{DISCUSSION AND CONCLUSIONS}

In this work, we have shown that spin-orbit coupling generates a characteristic field-angle dependence of the longitudinal thermal conductivity in nodal superconductors. These oscillations reflect how the density of states induced by a magnetic Zeeman field depends on the angle between the field and the spin polarization at the gap nodes. Although we have focused on the case of a $d_{x^{2}-y^{2}}$ superconductor with Rashba SOC, the mechanisms described in this work will be present for any nodal superconductor in which spin-orbit physics leads to spin-momentum locking at the gap nodes. At low temperature $(T \ll \mu B)$, the oscillation pattern is a simple function of the angle between the nodal spin axis and the magnetic field, and therefore depends both on the location of the nodes and the structure of the SOC. When the SOC is known, the magnetothermal oscillations can be used to determine the locations of the nodes; conversely, when the node positions are known, the oscillations yield a fingerprint of the SOC.

This analysis is simplest if there is a clean separation between spin-orbit and vortex contributions to the magnetothermal oscillations. Indeed, the field-angle oscillations are qualitatively similar in both cases, and the question of how one may distinguish them is important. For $d$-wave superconductors, it is common to write the field-angle dependence of the thermal conductivity as a series,

$$
\begin{aligned}
& \kappa_{x x}=\kappa_{0, x x}+\kappa_{2} \cos 2 \phi+\kappa_{4} \cos 4 \phi+\ldots, \\
& \kappa_{y y}=\kappa_{0, y y}-\kappa_{2} \cos 2 \phi+\kappa_{4} \cos 4 \phi+\ldots
\end{aligned}
$$


The terms $\kappa_{2}$ and $\kappa_{4}$ are the amplitudes of the twofold- and fourfold-symmetric contributions to $\kappa_{x x}$, respectively, and $\phi$ is the field angle as before. The fourfold term, $\kappa_{4}$, is a direct consequence of the fourfold symmetry of the excitation spectrum. It is generally attributed to the symmetry of the gap function, but may also reflect the underlying band structure [4].

$\kappa_{4}$ may be positive or negative, with $\kappa_{x x}$ having its minimum at $\phi=\pi / 4$ in the first case, and its maximum at $\pi / 4$ in the second. For oscillations due to the vortex lattice, the general trend at low fields is that $\kappa_{x x}$ is positive at low $T$, but then goes negative as $T$ is increased [3]. Figure 3 shows that the trend is similar here, with the crossover happening at $k_{B} T \approx 0.2 \mu B$ in the clean limit. The $T$ dependence of $\kappa_{4}$ therefore does not allow us to isolate the source of the oscillations. However, the field-strength dependence is qualitatively different for the two mechanisms, and does allow one to distinguish between them. In the clean limit $(\mu B \gg \gamma)$ and at low $T, \kappa_{4}$ grows as $\sqrt{B}$ for vortex-driven oscillations [2] but as $B$ for SOC-driven oscillations.

Another distinction between SOC- and vortex-driven magnetothermal conductivity oscillations lies in $\kappa_{2}$. In previous work, $\kappa_{2}$ was found to arise from the scattering of thermal currents by the vortices, and the twofold anisotropy reflects the difference between driving currents parallel to and perpendicular to the vortices. While in many materials this term is dominant at elevated temperatures, it is suppressed in quasi-2D materials where the circulating currents are small. Formally, $\kappa_{2}=0$ in our calculations, and any nonzero value of $\kappa_{2}$ would therefore signal a nonzero vortex contribution. A weak $\kappa_{2}$ in conjunction with a significant $\kappa_{4}$ is a strong hint that SOC is the dominant factor in observed magnetothermal conductivity oscillations.

One consequence of $\kappa_{2}$ vanishing is that, for the SOCdriven oscillations, the longitudinal thermal conductivity should be the same for both [100] and [110] heat currents provided the material is tetragonal, and provided the transverse components $\kappa_{x y}$ and $\kappa_{y x}$ vanish. Under typical experimental conditions the heat current perpendicular to the applied temperature gradient is zero, and the [100] and [110] longitudinal thermal conductivities are therefore [3]

$$
\kappa_{l}=\left\{\begin{array}{ll}
\kappa_{x x}-\frac{\kappa_{x y} \kappa_{y x}}{\kappa_{y y}}, & {[100]} \\
2 \frac{\kappa_{x x} \kappa_{y y}-\kappa_{x y} \kappa_{y x}}{\kappa_{x x}+\kappa_{y y}+\kappa_{x y}+\kappa_{y x}} & {[110]}
\end{array} .\right.
$$

For purely SOC-driven oscillations the intrinsic contribution to $\kappa_{x y} / T$ in the limit $T \rightarrow 0$ vanishes for a $d$-wave superconductor in an in-plane Zeeman field [49,52], and the leading-order intrinsic contribution to $\kappa_{x y}$ must therefore be $O\left(T^{2}\right)$. Since the leading-order contributions to $\kappa_{x x}$ and $\kappa_{y y}$ are $O(T), \lim _{T \rightarrow 0} \kappa_{l}$ reduces to $\kappa_{x x}$ and $2 \kappa_{x x} \kappa_{y y} /\left(\kappa_{x x}+\kappa_{y y}\right)$ for the [100] and [110] directions, respectively. These are equal for tetragonal superconductors.

For the case of $\mathrm{YBCO}_{6+x}$, we note that the material itself is orthorhombic due to the presence of one-dimensional $\mathrm{CuO}$ chains. This twofold anisotropy is tied to the crystal lattice, rather than the vortex lattice, and will show up primarily as a difference in the zeroth order term in the expansions of $\kappa_{x x}$ and $\kappa_{y y}$, so that $\kappa_{0, x x} \neq \kappa_{0, y y}$.

We can estimate the relative importance of the vortex and SOC contributions for $\mathrm{YBCO}_{6.5}$ by focusing on the specific heat. At this doping level, $\mathrm{YBCO}_{6+x}$ is strongly anisotropic, with the $c$-axis conductivity a factor of $10^{3}$ smaller than the in-plane conductivity. The DOS induced by circulating vortex currents can be obtained from the clean-limit $(\gamma \rightarrow 0)$ approximation given in Ref. [2],

$$
\rho(\phi) \approx \rho_{0} \frac{2 \sqrt{2} E_{H}}{\pi \hbar v_{2}} \max (|\sin \phi|,|\cos \phi|),
$$

with $\rho_{0}$ the normal state DOS and

$$
E_{H} \sim \frac{v_{F}}{2} \sqrt{\frac{\lambda_{a b}}{\lambda_{c}}} \sqrt{\frac{\pi B}{\Phi_{0}}},
$$

where $\Phi_{0}=\pi \hbar / e$ is the superconducting flux quantum. In $\mathrm{YBCO}_{6.5}$, the ratio of in-plane and out-of-plane penetration depths $\lambda_{c} / \lambda_{a b} \approx 35$ [58,59], and the fitted dispersion $\epsilon_{\mathbf{k}}$ gives the normal state DOS at the Fermi level $\rho_{0}=3.35 \mathrm{eV}^{-1}$. The resultant shift in the specific heat constant, $\Delta \gamma_{0}$ is shown in Fig. 2(c) and is considerably smaller than that obtained from SOC. It is reasonable to expect a similar disparity for the thermal conductivity, so that experimental observations of magnetothermal conductivity oscillations for in-plane fields, along with a small value for $\kappa_{2}$, would be consistent with significant spin-orbit coupling.

We finish with a few caveats. First, we note that a detailed quantitative description of any particular material will depend on the details of the scattering rate. In particular, we have taken a simple model in which $\gamma$ depends on neither wave vector or energy. While such an assumption is sensible for many materials, it can be problematic in nodal superconductors where the scattering rate can have a nontrivial energy dependence that is determined by the strength of the scattering potential. In cuprates, for example, quantitatively accurate models typically require an admixture of Born and unitary scatterers [60-62]. Each of these has a characteristic energy dependence that will modify the temperature dependence of the oscillations. Rigorous modeling of experiments will thus require a realistic disorder model.

Second, the question of how to extract the size of the spinorbit coupling constant $\alpha$ remains open. While the existence of SOC in a nodal superconductor is easy to establish via the thermal conductivity oscillations, the amplitude of the spin-orbit coupling constant is harder to determine. In clean materials, the size of the oscillations is nearly independent of $\alpha$ [Fig. 5(a)] and there is no clear crossover in behavior as a function of magnetic field strength between $\mu B \ll \alpha$ and $\mu B \gg \alpha$. Rather, the amplitude of the oscillations is an indication of whether the spin splitting of the Fermi surfaces is large or small relative to the scattering rate.

Third, we have neglected in this work variations of the chemical potential with the field angle, which is another potential contribution to the field-angle dependence of $\kappa_{x x}$. In our analytical calculations, these variations would manifest themselves as modulations of both the Fermi velocity and the anomalous velocity, $v_{2}$. We have checked numerically that, at least for the model used in this work, chemical potential modulations have a negligible effect on the thermal conductivity.

In summary, we have demonstrated the existence of a novel intrinsic mechanism for oscillations of the longitudinal magnetothermal conductivity as a function of magnetic field angle. 
Zeeman coupling to nodal quasiparticles inflates the nodes into Bogoliubov Fermi surfaces, whose sizes depend on the field angle, the structure of the SOC, and the location of the gap nodes. The magnitude of the induced specific heat and thermal conductivity both depend on the sizes of the induced Fermi surfaces, and the angle dependence of the thermal properties provides a tool to explore the SOC (if the locations of the gap nodes are known) or the nodal structure (if the SOC is known). As a probe of SOC, this technique has the advantage of being a bulk measurement, and is therefore insensitive to inversion-symmetry breaking at surfaces.

The structure of the magnetothermal oscillations is qualitatively similar to what was found earlier for vortex-induced oscillations, but can be distinguished by details of the field and angle dependence. Interestingly, we find that the pattern of the oscillations inverts at high temperature, similar to what was reported earlier for vortex-induced oscillations.

\section{ACKNOWLEDGMENTS}

W.A.A. acknowledges support by the Natural Sciences and Engineering Research Council (NSERC) of Canada. A.P.K. acknowledges support by the Deutsche Forschungsgemeinschaft (DFG, German Research Foundation)- project-ID107745057-TRR 80.

\section{APPENDIX A: SUPERCONDUCTING STATE}

We consider the superconducting state of a single $\mathrm{CuO}_{2}$ layer with Rashba SOC. The superconducting state is predominantly singlet, with a $d$-wave symmetry, but additional triplet pieces are induced by both the SOC and the in-plane Zeeman field. The goal of this section is to evaluate the size of the triplet components.

Taking the basis

$$
\mathbf{C}_{\mathbf{k}}=\left[\begin{array}{cccc}
c_{\mathbf{k} \uparrow}, & c_{\mathbf{k} \downarrow}, & c_{-\mathbf{k} \uparrow}^{\dagger}, & c_{-\mathbf{k} \downarrow}^{\dagger}
\end{array}\right]^{T},
$$

the Hamiltonian has the form $\hat{H}=\sum_{\mathbf{k}}{ }^{\prime} \mathbf{C}_{\mathbf{k}}^{\dagger} \mathbf{H}_{\mathbf{k}} \mathbf{C}_{\mathbf{k}}$, where the primed sum is restricted to half of the Brillouin zone, and

$$
\mathbf{H}_{\mathbf{k}}=\left[\begin{array}{cc}
\mathbf{h}_{\mathbf{k}} & \boldsymbol{\Delta}_{\mathbf{k}} \\
\boldsymbol{\Delta}_{\mathbf{k}}^{\dagger} & -\mathbf{h}_{\mathbf{k}}^{T}
\end{array}\right]
$$

with

$$
\mathbf{h}_{\mathbf{k}}=\left[\begin{array}{ll}
\epsilon_{\mathbf{k}} & \tilde{g}_{\mathbf{k}} \\
\tilde{g}_{\mathbf{k}}^{*} & \epsilon_{\mathbf{k}}
\end{array}\right]
$$

and

$$
\boldsymbol{\Delta}_{\mathbf{k}}=\left[\begin{array}{cc}
-d_{x \mathbf{k}}+i d_{y \mathbf{k}} & d_{z \mathbf{k}}+\chi_{d \mathbf{k}} \\
d_{z \mathbf{k}}-\chi_{d \mathbf{k}} & d_{x \mathbf{k}}+i d_{y \mathbf{k}}
\end{array}\right] .
$$

where $\tilde{g}_{\mathbf{k}}=\alpha\left(\sin k_{y}+i \sin k_{x}\right)-\mu B e^{-i \phi}$ and $\phi$ is the angle between the $x$ axis and the in-plane magnetic field.

We take the simplest form of pairing interaction appropriate for the cuprates,

$$
\begin{aligned}
V\left(\mathbf{k}-\mathbf{k}^{\prime}\right) & =V_{0}\left[\cos \left(k_{x}-k_{x}^{\prime}\right)+\cos \left(k_{y}-k_{y}^{\prime}\right)\right] \\
& =V_{0} \sum_{i} \eta_{i \mathbf{k}} \eta_{i \mathbf{k}^{\prime}}
\end{aligned}
$$

with

$$
\begin{aligned}
\eta_{d \mathbf{k}} & =\frac{1}{\sqrt{2}}\left(\cos k_{x}-\cos k_{y}\right), \\
\eta_{s \mathbf{k}} & =\frac{1}{\sqrt{2}}\left(\cos k_{x}+\cos k_{y}\right), \\
\eta_{x \mathbf{k}} & =\sin k_{x}, \\
\eta_{y \mathbf{k}} & =\sin k_{y} .
\end{aligned}
$$

Under the assumption that the singlet order parameter is $d$-wave, we write $\chi_{d \mathbf{k}}=\chi_{d} \eta_{d \mathbf{k}}$ with

$$
\chi_{d}=\frac{V_{0}}{4} \sum_{\mathbf{k}^{\prime}} \eta_{d \mathbf{k}^{\prime}}\left(\left\langle c_{-\mathbf{k}^{\prime} \downarrow} c_{\mathbf{k}^{\prime} \uparrow}\right\rangle-\left\langle c_{-\mathbf{k}^{\prime} \uparrow} c_{\mathbf{k}^{\prime} \downarrow}\right\rangle\right) .
$$

Similarly, the triplet components have odd spatial parity, and can therefore be written

$$
d_{x \mathbf{k}}=d_{x x} \eta_{x \mathbf{k}}+d_{x y} \eta_{y \mathbf{k}}, \text { etc. }
$$

with $(a=x, y)$

$$
\begin{aligned}
& d_{x a}=\frac{V_{0}}{4} \sum_{\mathbf{k}^{\prime}} \eta_{a \mathbf{k}^{\prime}}\left(\left\langle c_{-\mathbf{k}^{\prime} \downarrow} c_{\mathbf{k}^{\prime} \downarrow}\right\rangle-\left\langle c_{-\mathbf{k}^{\prime} \uparrow} c_{\mathbf{k}^{\prime} \uparrow}\right\rangle\right), \\
& d_{y a}=\frac{V_{0}}{4 i} \sum_{\mathbf{k}^{\prime}} \eta_{a \mathbf{k}^{\prime}}\left(\left\langle c_{-\mathbf{k}^{\prime} \downarrow} c_{\mathbf{k}^{\prime} \downarrow}\right\rangle+\left\langle c_{-\mathbf{k}^{\prime} \uparrow} c_{\mathbf{k}^{\prime} \uparrow}\right\rangle\right), \\
& d_{z a}=\frac{V_{0}}{4} \sum_{\mathbf{k}^{\prime}} \eta_{a \mathbf{k}^{\prime}}\left(\left\langle c_{-\mathbf{k}^{\prime} \downarrow} c_{\mathbf{k}^{\prime} \uparrow}\right\rangle+\left\langle c_{-\mathbf{k}^{\prime} \uparrow} c_{\mathbf{k}^{\prime} \downarrow}\right\rangle\right) .
\end{aligned}
$$

We choose parameters such that the singlet component of the order parameter is $\chi_{d}=50 \mathrm{meV}$, which is comparable to the antinodal gap in underdoped $\mathrm{YBCO}_{6+x}$.

SOC induces a triplet component of the form $\pm d_{x \mathbf{k}}+i d_{y \mathbf{k}}$ that goes along with the singlet piece [63]. For our model parameters, self-consistent calculations find that the triplet component is approximately $1 \%$ of the dominant singlet component, and is nearly independent of magnetic field strength and direction. In addition, the Zeeman field induces a second triplet component $d_{z \mathbf{k}}$ that depends on field angle. This component is found to be three orders of magnitude smaller than the singlet component.

\section{APPENDIX B: THERMAL CONDUCTIVITY: LARGE SOC LIMIT}

In this Appendix, we derive an analytic approximation for $\Pi^{x x}(x)$ that is valid in the limit $\alpha \gg \gamma, \mu B$. To evaluate Eq. (16), we transform both the Hamiltonian and the quasiparticle velocity operators to the helical basis. When the SOC is large, we can neglect the mixing of bands of different helicities, either by impurities or by the magnetic field. This simplification allows us to derive an explicit expression for $\Pi^{x x}(x)$.

a. The Hamiltonian in the helical basis. As a first step, we transform the Hamiltonian, Eq. (A2), to the helical basis via the unitary transformation,

$$
\mathcal{U}_{\mathbf{k}}=\left[\begin{array}{cc}
\mathbf{U}_{\mathbf{k}} & 0 \\
0 & \mathbf{U}_{-\mathbf{k}}^{*}
\end{array}\right] ; \quad \mathbf{U}_{\mathbf{k}}=\frac{1}{\sqrt{2}}\left[\begin{array}{cc}
1 & -e^{i \theta_{\mathbf{k}}} \\
e^{-i \theta_{\mathbf{k}}} & 1
\end{array}\right],
$$


with $e^{i \theta_{\mathbf{k}}}=g_{\mathbf{k}} /\left|g_{\mathbf{k}}\right|$ and $g_{\mathbf{k}}=\alpha\left(\sin k_{y}+i \sin k_{x}\right)$. In zero field, $\mathbf{U}_{\mathbf{k}}$ diagonalizes the nonsuperconducting Hamiltonian $\mathbf{h}_{\mathbf{k}}$, with $\mathbf{U}_{\mathbf{k}}^{\dagger} \mathbf{h}_{\mathbf{k}} \mathbf{U}_{\mathbf{k}}=\operatorname{diag}\left(\xi_{\mathbf{k}+}, \xi_{\mathbf{k}-}\right)$ and $\xi_{\mathbf{k} \pm}=\epsilon_{\mathbf{k}} \pm\left|g_{\mathbf{k}}\right|$.

In the superconducting state and with nonzero in-plane magnetic field, we obtain the transformed Hamiltonian,

$$
\mathbf{H}_{\mathbf{k}}^{\xi}=\mathcal{U}_{\mathbf{k}}^{\dagger} \mathbf{H}_{\mathbf{k}} \mathcal{U}_{\mathbf{k}}=\left[\begin{array}{cc}
\mathbf{h}_{\mathbf{k}}^{\xi} & \boldsymbol{\Delta}_{\mathbf{k}}^{\xi} \\
\boldsymbol{\Delta}_{\mathbf{k}}^{\dagger \dagger} & -\mathbf{h}_{-\mathbf{k}}^{\xi}
\end{array}\right]
$$

The diagonal block is

$$
\mathbf{h}_{\mathbf{k}}^{\xi}=\left[\begin{array}{cc}
\xi_{\mathbf{k}+}-\mu B \cos \left(\theta_{\mathbf{k}}+\phi\right) & i \mu B e^{i \theta_{\mathbf{k}}} \sin \left(\theta_{\mathbf{k}}+\phi\right) \\
-i \mu B e^{-i \theta_{\mathbf{k}}} \sin \left(\theta_{\mathbf{k}}+\phi\right) & \xi_{\mathbf{k}-}+\mu B \cos \left(\theta_{\mathbf{k}}+\phi\right)
\end{array}\right] .
$$

The off-diagonal block has a similar structure to Eq. (A4); however, it simplifies considerably if the triplet components of the order parameter can be neglected. Then

$$
\Delta_{\mathbf{k}}^{\xi}=\left[\begin{array}{cc}
\Delta_{\mathbf{k}}^{+} & 0 \\
0 & \Delta_{\mathbf{k}}^{-}
\end{array}\right]
$$

with $\Delta_{\mathbf{k}}^{ \pm}=-e^{ \pm i \theta_{\mathbf{k}}} \chi_{d \mathbf{k}}$. When $B=0$, the two helical bands are not mixed by singlet superconductivity under the restriction of zero-momentum pairing, and the Hamiltonian $\mathbf{H}_{\mathbf{k}}^{\xi}$ thus describes two independent superconducting bands, each of which has a BCS-like structure.

$b$. The velocity matrix in the helical basis.. Next, we transform the matrix defined by Eq. (14) via

$$
\mathbf{V}_{\mathbf{k}}^{\xi}=\mathcal{U}_{\mathbf{k}}^{\dagger} \mathbf{V}_{\mathbf{k}} \mathcal{U}_{\mathbf{k}}
$$

The top left block transforms as

$$
\mathbf{U}_{\mathbf{k}}^{\dagger} \mathbf{v}_{\mathbf{k}} \mathbf{U}_{\mathbf{k}}=\left[\begin{array}{cc}
\nabla_{\mathbf{k}} \xi_{\mathbf{k}}^{+} & 0 \\
0 & \nabla_{\mathbf{k}} \xi_{\mathbf{k}}^{-}
\end{array}\right]+i\left(\nabla_{\mathbf{k}} \theta_{\mathbf{k}}\right)\left[\begin{array}{cc}
0 & g_{\mathbf{k}} \\
-g_{\mathbf{k}}^{*} & 0
\end{array}\right],
$$

while the top right block is

$$
\mathbf{U}_{\mathbf{k}}^{\dagger} \mathbf{v}_{\Delta, \mathbf{k}} \mathbf{U}_{-\mathbf{k}}^{*}=\left[\begin{array}{cc}
-e^{i \theta_{\mathbf{k}}} \nabla_{\mathbf{k}} \chi_{d \mathbf{k}} & 0 \\
0 & -e^{-i \theta_{\mathbf{k}}} \nabla_{\mathbf{k}} \chi_{d \mathbf{k}}
\end{array}\right]
$$

Then

$$
\mathbf{V}_{\mathbf{k}}^{\xi}=\left[\begin{array}{cccc}
\mathbf{v}_{\mathbf{k}+}^{\xi} & \mathbf{v}_{\mathbf{k}}^{\mathrm{an}} & \mathbf{v}_{\Delta, \mathbf{k}}^{+} & 0 \\
\left(\mathbf{v}_{\mathbf{k}}^{\mathrm{an}}\right)^{*} & \mathbf{v}_{\mathbf{k}-}^{\xi} & 0 & \mathbf{v}_{\Delta, \mathbf{k}}^{-} \\
\left(\mathbf{v}_{\Delta, \mathbf{k}}^{+}\right)^{*} & 0 & \mathbf{v}_{-\mathbf{k}+}^{\xi} & \left(\mathbf{v}_{-\mathbf{k}}^{\mathrm{an}}\right)^{*} \\
0 & \left(\mathbf{v}_{\Delta, \mathbf{k}}^{-}\right)^{*} & \mathbf{v}_{-\mathbf{k}}^{\mathrm{an}} & \mathbf{v}_{-\mathbf{k}-}^{\xi}
\end{array}\right]
$$

where $\mathbf{v}_{\mathbf{k} \alpha}^{\xi}=\nabla_{\mathbf{k}} \xi_{\mathbf{k} \alpha}, \quad \mathbf{v}_{\Delta, \mathbf{k}}^{a}=-e^{i a \theta_{\mathbf{k}}} \nabla_{\mathbf{k}} \chi_{d \mathbf{k}}$, and the SOCrelated anomalous velocity is $\mathbf{v}_{\mathbf{k}}^{\text {an }}=i\left(\nabla_{k} \theta_{\mathbf{k}}\right) g_{\mathbf{k}}$.

c. Reordering the basis. To move forward, it is useful to rearrange the Hamiltonian and velocity into blocks with given helicity,

$$
\left[\begin{array}{c}
\psi_{\mathbf{k}+} \\
\psi_{\mathbf{k}-} \\
\psi_{-\mathbf{k}+}^{\dagger} \\
\psi_{-\mathbf{k}-}^{\dagger}
\end{array}\right] \rightarrow\left[\begin{array}{c}
\psi_{\mathbf{k}+} \\
\psi_{-\mathbf{k}+}^{\dagger} \\
\psi_{\mathbf{k}-} \\
\psi_{-\mathbf{k}-}^{\dagger}
\end{array}\right]
$$

The velocity operator becomes

$$
\begin{aligned}
\mathbf{V}_{\mathbf{k}}^{\xi} & =\left[\begin{array}{cc|cc}
\mathbf{v}_{\mathbf{k}+}^{\xi} & \mathbf{v}_{\Delta, \mathbf{k}}^{+} & \mathbf{v}_{\mathbf{k}}^{\mathrm{an}} & 0 \\
\left(\mathbf{v}_{\Delta, \mathbf{k}}^{+}\right)^{*} & \mathbf{v}_{-\mathbf{k}+}^{\xi} & 0 & \left(\mathbf{v}_{-\mathbf{k}}^{\mathrm{an}}\right)^{*} \\
\hline\left(\mathbf{v}_{\mathbf{k}}^{\mathrm{an}}\right)^{*} & 0 & \mathbf{v}_{\mathbf{k}-}^{\xi} & \mathbf{v}_{\Delta, \mathbf{k}}^{-} \\
0 & \mathbf{v}_{-\mathbf{k}}^{\mathrm{an}} & \left(\mathbf{v}_{\Delta, \mathbf{k}}^{-}\right)^{*} & \mathbf{v}_{-\mathbf{k}-}^{\xi}
\end{array}\right] \\
& =\mathbf{V}_{\mathbf{k}}^{0}+\mathbf{V}_{\mathbf{k}}^{\mathrm{an}},
\end{aligned}
$$

where $\mathbf{V}_{\mathbf{k}}^{0}$ contains the diagonal blocks of $\mathbf{V}_{\mathbf{k}}^{\xi}$ and $\mathbf{V}_{\mathbf{k}}^{\text {an }}$ contains the off-diagonal blocks. The Hamiltonian is

$$
\mathbf{H}_{\mathbf{k}}^{\xi}=\left[\begin{array}{cc}
\mathbf{h}_{\mathbf{k}+} & \mathbf{h}_{\mathbf{k}}^{\prime} \\
\mathbf{h}_{\mathbf{k}}^{\prime} & \mathbf{h}_{\mathbf{k}-}
\end{array}\right]
$$

with

$\mathbf{h}_{\mathbf{k} a}=\left[\begin{array}{cc}\xi_{\mathbf{k} a}-a \mu B \cos \left(\theta_{\mathbf{k}}+\phi\right) & \Delta_{\mathbf{k}}^{a} \\ \Delta_{\mathbf{k}}^{a *} & -\xi_{\mathbf{k} a}-a \mu B \cos \left(\theta_{\mathbf{k}}+\phi\right)\end{array}\right]$,

where $a= \pm$ labels the band helicity and

$$
\mathbf{h}_{\mathbf{k}}^{\prime}=i \mu B \sin \left(\theta_{\mathbf{k}}+\phi\right)\left[\begin{array}{cc}
e^{-i \theta_{\mathbf{k}}} & 0 \\
0 & e^{i \theta_{\mathbf{k}}}
\end{array}\right] .
$$

The matrix $\mathbf{h}_{\mathbf{k}}^{\prime}$ mixes bands with different helicities and may be dropped when $\mu B \ll|\alpha|$.

d. Spectral function. To zeroth order in $\mathbf{h}_{\mathbf{k}}^{\prime}, \mathbf{H}_{\mathbf{k}}^{\xi}$ has the energy eigenvalues

$$
\begin{aligned}
E_{\mathbf{k} a s} & =-a \mu B \cos \left(\theta_{\mathbf{k}}+\phi\right)+s E_{\mathbf{k} a}, \\
E_{\mathbf{k} a} & =\sqrt{\xi_{\mathbf{k} a}^{2}+\left|\Delta_{\mathbf{k} a}\right|^{2}}
\end{aligned}
$$

where $a= \pm$ is the helicity index and $s= \pm$ indicates whether the quasiparticle branch is upward or downward dispersing. The Green's function is

$$
\mathbf{g}_{a b}^{0}(\mathbf{k}, z)=\frac{\delta_{a, b}}{\tilde{z}_{a}^{2}-E_{\mathbf{k} a}^{2}}\left[\begin{array}{cc}
\tilde{z}_{a}+\xi_{\mathbf{k} a} & \Delta_{\mathbf{k} a} \\
\Delta_{\mathbf{k} a}^{*} & \tilde{z}_{a}-\xi_{\mathbf{k} a}
\end{array}\right],
$$

where $a$ and $b$ represent the different helicities, and $\tilde{z}_{ \pm}=$ $z \pm \mu B \cos \left(\theta_{\mathbf{k}}+\phi\right)$ with $z$ a complex frequency. To obtain the spectral function for (real) frequency $x$, we take

$$
\begin{aligned}
\mathbf{a}_{a b}^{0}(\mathbf{k}, x)= & \frac{1}{2 \pi i}\left[\mathbf{g}_{a b}^{0}(\mathbf{k}, x-i \gamma)-\mathbf{g}_{a b}^{0}(\mathbf{k}, x+i \gamma)\right] \\
= & \frac{\delta_{a, b}}{2 E_{\mathbf{k} a}} \sum_{ \pm} \pm \delta\left( \pm E_{\mathbf{k} a}-x-a \mu B \cos \left(\theta_{\mathbf{k}}+\phi\right)\right) \\
& \times\left[\begin{array}{cc}
\xi_{\mathbf{k} a} \pm E_{\mathbf{k} a} & \Delta_{\mathbf{k} a} \\
\Delta_{\mathbf{k} a}^{*} & -\xi_{\mathbf{k} a} \pm E_{\mathbf{k} a}
\end{array}\right] .
\end{aligned}
$$

In this expression, the $\delta$ functions are understood as Lorentzians, $\delta(x)=\pi^{-1} \gamma /\left(x^{2}+\gamma^{2}\right)$.

e. Thermal conductivity. We evaluate the transport kernel $\Pi(x)$ from Eq. (16), using both the leading-order spectral function, Eq. (B18), and the leading-order velocity operator $\mathbf{V}_{\mathbf{k}}^{0}$ defined in Eq. (B11), which neglects interband mixing due to the anomalous velocity term $\mathbf{v}_{\mathbf{k}}^{\text {an }}$. As noted before, this calculation is zeroth order in interband mixing, but not zeroth order in the magnetic field. 

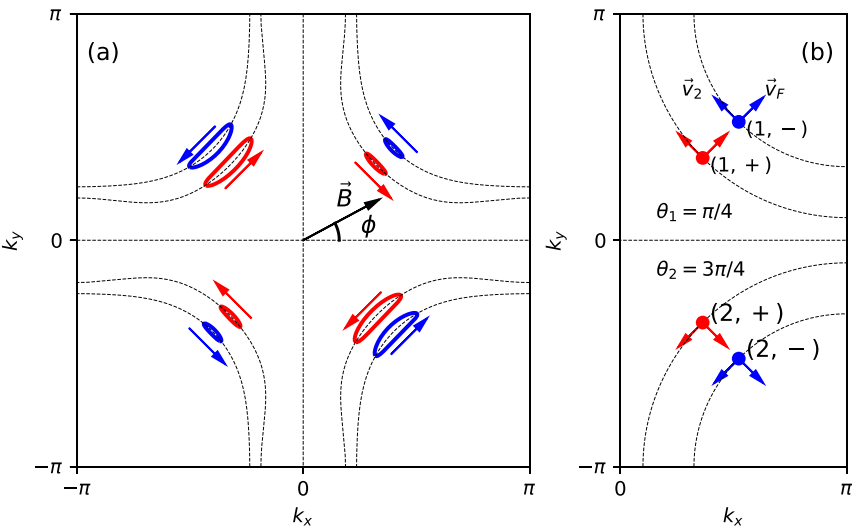

FIG. 7. (a) Bogoliubov Fermi surface pockets in the full Brillouin zone, along with the spin polarization of each quasiparticle branch near the node. (b) Schematic of the reduced Brillouin zone, which consists of the region $0<k_{x}<\pi,-\pi<k_{y}<\pi$. Nodal points, at which the superconducting $d$-wave order parameter vanishes, are indicated by dots. Arrows located at each dot indicate the directions of the normal and superconducting group velocities. Local coordinate axes $k_{1}$ and $k_{2}$ are attached to each of the nodes, and are parallel to the velocities. Each of the four nodes is labeled by its quadrant $(n=1,2)$ and its helicity $(a= \pm)$.

We take the Brillouin zone to be $0<k_{x}<\pi,-\pi<k_{y}<$ $\pi$, and sum over four nodal regions [Fig. 7(b)]; two of these correspond to a sum over helicty index $a$; the other two will be indicated by a sum over nodal index $n=1,2$. We use rotated momenta $k_{1}$ and $k_{2}$ near each node, with the understanding that they are rotated by $90^{\circ}$ between nodes in regions 1 and 2 , and that the zero of the coordinate system is at the nodal point for each $(n, a)$. The advantage of this definition is that we can write

$$
\xi_{\mathbf{k} a}=\hbar v_{F} k_{1} ; \quad \Delta_{\mathbf{k} a}=e^{i a \theta_{n}} \hbar v_{2} k_{2},
$$

for each node, with the approximation that $\theta_{\mathbf{k}}$ can be treated as constant in the neighborhood of each node. Recalling that $\tan \theta_{\mathbf{k}}=\sin k_{x} / \sin k_{y}$, we obtain $\theta_{1}=\pi / 4$ for node 1 and $\theta_{2}=3 \pi / 4$ for node 2 . Similarly, we will assume that the quasiparticle velocities depend only on the nodal index $n$, and are constant in the vicinity of each node.

Under these transformations,

$$
\begin{aligned}
\frac{1}{N_{k}} \sum_{\mathbf{k}}^{\prime} & \rightarrow \frac{a_{0}^{2}}{2 \pi^{2}} \int d k_{x} d k_{y} \\
& \rightarrow \frac{a_{0}^{2}}{2 \pi^{2} \hbar^{2} v_{F} v_{2}} \sum_{n=1}^{2} \sum_{a= \pm} \int_{0}^{\infty} E d E \int_{0}^{2 \pi} d \zeta,
\end{aligned}
$$

where $a_{0}$ is the lattice constant. The kernel for the thermal conductivity is therefore

$$
\begin{aligned}
\Pi^{x x}(x)= & \frac{1}{4 \pi^{2}} \sum_{a= \pm} \sum_{n=1}^{2} \int_{0}^{\infty} \frac{E d E}{v_{F} v_{2}} \oint d \zeta \\
& \times \operatorname{Tr}\left[\mathbf{a}_{n, a a}^{0}(E, \zeta, x) \mathbf{v}_{n, a a}^{x} \mathbf{a}_{n, a a}^{0}(E, \zeta, x) \mathbf{v}_{n, a a}^{x}\right]
\end{aligned}
$$

where we have made the linearized nodal approximation, $\xi=E \cos \zeta, \Delta_{a}=E e^{i a \theta_{n}} \sin \zeta$ and $\zeta \in[0,2 \pi]$. The trace in these equations is over particle-hole channels associated with superconductivity, so the matrices are $2 \times 2$. The velocity matrix $\mathbf{v}_{n, a a}^{x}$ is the $2 \times 2$ matrix obtained from the top-left $(a=+)$ or bottom-right $(a=-)$ block of $\mathbf{V}_{\mathbf{k}}^{0}$. The superscript $x$ refers to the component of the quasiparticle velocity along the $x$ direction. Noting that $v_{F}^{x}=v_{F} / \sqrt{2}$ and $v_{2}^{x}=-v_{2} / \sqrt{2}$ [Fig. 7(b)],

$$
\mathbf{v}_{n, a a}^{x}=\frac{1}{\sqrt{2}}\left[\begin{array}{cc}
v_{F} & -e^{i a \theta_{n}} v_{2} \\
-e^{-i a \theta_{n}} v_{2} & -v_{F}
\end{array}\right] .
$$

In linearized nodal coordinates, the spectral function in Eq. (B22) is

$$
\begin{aligned}
\mathbf{a}_{n, a a}^{0}(E, \zeta, x)= & \frac{1}{2} \sum_{s= \pm} \delta\left[s E-x-a \mu B \cos \left(\theta_{n}+\phi\right)\right] \\
& \times\left[\begin{array}{cc}
1+s \cos \zeta & s e^{i a \theta_{n}} \sin \zeta \\
s e^{-i a \theta_{n}} \sin \zeta & 1-s \cos \zeta
\end{array}\right] .
\end{aligned}
$$

Separating the thermal conductivity kernel into contributions from the two helicity bands, $\Pi^{x x}(x)=\sum_{a} \Pi^{a}(x)$, we obtain for the + helicity band

$$
\begin{aligned}
\Pi^{+}(x)= & \frac{1}{4 \pi^{2} v_{F} v_{2}}\left\{\int_{0}^{\infty} d E E \delta^{2}\left[E-x-\mu B \cos \left(\theta_{1}+\phi\right)\right]\right\} \\
& \times \frac{1}{8} \oint d \zeta \operatorname{Tr}\left[\left[\begin{array}{cc}
1+\cos \zeta & e^{i \theta_{1}} \sin \zeta \\
e^{-i \theta_{1}} \sin \zeta & 1-\cos \zeta
\end{array}\right]\left[\begin{array}{cc}
v_{F} & -e^{i \theta_{1}} v_{2} \\
-e^{-i \theta_{1}} v_{2} & -v_{F}
\end{array}\right]\left[\begin{array}{cc}
1+\cos \zeta & e^{i \theta_{1}} \sin \zeta \\
e^{-i \theta_{1}} \sin \zeta & 1-\cos \zeta
\end{array}\right]\left[\begin{array}{cc}
v_{F} & -e^{i \theta_{1}} v_{2} \\
-e^{-i \theta_{1}} v_{2} & -v_{F}
\end{array}\right]\right] \\
& +\frac{1}{4 \pi^{2} v_{F} v_{2}}\left\{\int_{0}^{\infty} d E E \delta^{2}\left[E+x+\mu B \cos \left(\theta_{1}+\phi\right)\right]\right\} \\
& \times \frac{1}{8} \oint d \zeta \operatorname{Tr}\left[\left[\begin{array}{cc}
1-\cos \zeta & -e^{i \theta_{1}} \sin \zeta \\
-e^{-i \theta_{1}} \sin \zeta & 1+\cos \zeta
\end{array}\right]\left[\begin{array}{cc}
v_{F} & -e^{i \theta_{1}} v_{2} \\
-e^{-i \theta_{1}} v_{2} & -v_{F}
\end{array}\right]\left[\begin{array}{cc}
1-\cos \zeta & -e^{i \theta_{1}} \sin \zeta \\
-e^{-i \theta_{1}} \sin \zeta & 1+\cos \zeta
\end{array}\right]\left[\begin{array}{cc}
v_{F} & -e^{i \theta_{1}} v_{2} \\
-e^{-i \theta_{1}} v_{2} & -v_{F}
\end{array}\right]\right] \\
& +\frac{2}{4 \pi^{2} v_{F} v_{2}}\left\{\int_{0}^{\infty} d E E \delta\left[E-x-\mu B \cos \left(\theta_{1}+\phi\right)\right] \delta\left[E+x+\mu B \cos \left(\theta_{1}+\phi\right)\right]\right\} \\
& \times \frac{1}{8} \oint d \zeta \operatorname{Tr}\left[\left[\begin{array}{ccc}
1+\cos \zeta & e^{i \theta_{1}} \sin \zeta \\
e^{-i \theta_{1}} \sin \zeta & 1-\cos \zeta
\end{array}\right]\left[\begin{array}{ccc}
v_{F} & -e^{i \theta_{1}} v_{2} \\
-e^{-i \theta_{1}} v_{2} & -v_{F}
\end{array}\right]\left[\begin{array}{cc}
1-\cos \zeta & -e^{i \theta_{1}} \sin \zeta \\
-e^{-i \theta_{1}} \sin \zeta & 1+\cos \zeta
\end{array}\right]\left[\begin{array}{cc}
v_{F} & -e^{i \theta_{1}} v_{2} \\
-e^{-i \theta_{1}} v_{2} & -v_{F}
\end{array}\right]\right] \\
& +\left(\theta_{1} \rightarrow \theta_{2}\right)
\end{aligned}
$$


Using the Lorentzian form for the delta function, the energy integrals easily give

$$
\begin{aligned}
\frac{\gamma^{2}}{\pi^{2}} \int_{0}^{\infty} d E \frac{E}{\left[(E \mp \tilde{x})^{2}+\gamma^{2}\right]^{2}} & =\frac{1}{2 \pi^{2}}\left[1 \pm \frac{\tilde{x}}{\gamma}\left(\frac{\pi}{2} \pm \tan ^{-1} \frac{\tilde{x}}{\gamma}\right)\right], \\
\frac{\gamma^{2}}{\pi^{2}} \int_{0}^{\infty} d E \frac{E}{\left[(E-\tilde{x})^{2}+\gamma^{2}\right]\left[(E+\tilde{x})^{2}+\gamma^{2}\right]} & =\frac{1}{2 \pi^{2}} \frac{\gamma}{\tilde{x}} \tan ^{-1} \frac{\tilde{x}}{\gamma} .
\end{aligned}
$$

Performing the matrix multiplications, taking the traces, and integrating over the angle $\zeta$ gives

$$
\Pi^{+}(x)=\frac{\left(v_{F}^{2}+v_{2}^{2}\right)}{8 \pi^{3} v_{F} v_{2}}\left\{2+\mathcal{E}\left[\frac{x+\mu B \cos \left(\frac{\pi}{4}+\phi\right)}{\gamma}\right]+\mathcal{E}\left[\frac{x+\mu B \cos \left(\frac{3 \pi}{4}+\phi\right)}{\gamma}\right]\right\},
$$

where

$$
\mathcal{E}(x)=\left(x+\frac{1}{x}\right) \tan ^{-1} x .
$$

A nearly identical calculation gives the contribution $\Pi^{-}(x)$ to the thermal conductivity kernel from the - helicity bands. $\Pi^{-}(x)$ has the same form as Eq. (B28), but with $\mu B \rightarrow-\mu B$. The total kernel is then

$$
\Pi^{x x}(x)=\frac{1}{8 \pi^{3}} \frac{v_{F}^{2}+v_{2}^{2}}{v_{F} v_{2}}\left\{4+\sum_{ \pm} \mathcal{E}\left[\frac{x \pm \mu B \cos \left(\frac{\pi}{4}+\phi\right)}{\gamma}\right]+\sum_{ \pm} \mathcal{E}\left[\frac{x \pm \mu B \cos \left(\frac{3 \pi}{4}+\phi\right)}{\gamma}\right]\right\}
$$

The thermal conductivity follows from

$$
\frac{\kappa_{x x}}{T}=\frac{\pi}{\hbar d T^{2}} \int d x x^{2} \frac{\partial f(x)}{\partial x} \Pi^{x x}(x) .
$$

In the limit $T \rightarrow 0$, this expression simplifies to

$$
\frac{\kappa_{x x}}{T} \rightarrow \frac{k_{B}^{2} \pi^{3}}{3 \hbar d} \Pi^{x x}(0)=\frac{k_{B}^{2}}{12 \hbar d} \frac{v_{F}^{2}+v_{2}^{2}}{v_{F} v_{2}}\left\{2+\mathcal{E}\left[\frac{\mu B \cos \left(\frac{\pi}{4}+\phi\right)}{\gamma}\right]+\mathcal{E}\left[\frac{\mu B \cos \left(\frac{3 \pi}{4}+\phi\right)}{\gamma}\right]\right\} .
$$

As an important check, notice that as $\mu B \rightarrow 0, \mathcal{E} \rightarrow 1$, and

$$
\frac{\kappa_{x x}^{0}}{T} \rightarrow \frac{k_{B}^{2}}{3 \hbar d} \frac{v_{F}^{2}+v_{2}^{2}}{v_{F} v_{2}}
$$

which is the result first worked out by Durst and Lee [51].

\section{APPENDIX C: DENSITY OF STATES: LARGE SOC LIMIT}

In this section, we derive an expression for the DOS induced by the in-plane magnetic field in the limit of large SOC, namely $|\alpha| \gg \mu B, \gamma$.

From Eq. (B18), the spectral function is approximately

$$
\mathbf{a}_{a a}^{0}(\mathbf{k}, x)=\frac{1}{2} \sum_{s= \pm 1} \delta\left[s E_{\mathbf{k} a}-x-a \mu B \cos \left(\theta_{\mathbf{k}}+\phi\right)\right]\left[\begin{array}{cc}
1+s \cos \zeta & s \sin \zeta \\
s \sin \zeta & 1-s \cos \zeta
\end{array}\right] .
$$

For the residual DOS at the Fermi energy, we set $x=0$ and sum k over the reduced Brillouin zone [Fig. 7(b)]. Since both helicities make identical contributions to the DOS, we calculate the result for the positive helicity and multiply the result by 2 . Making use of Eq. (B21), we obtain

$$
\rho\left(\varepsilon_{F}\right)=\frac{2}{N_{k}} \sum_{\mathbf{k}}^{\prime}\left[\mathbf{a}_{++}^{0}(\mathbf{k}, 0)\right]_{11}=\frac{a_{0}^{2}}{2 \pi^{2} v_{F} v_{2}} \sum_{n=1}^{2} \sum_{s= \pm} \oint d \zeta \int_{0}^{\infty} E d E \delta\left[s E+\mu B \cos \left(\theta_{n}+\phi\right)\right],
$$

where $\theta_{1}=\frac{\pi}{4}$ and $\theta_{2}=\frac{3 \pi}{4}$. Again, substituting Lorentzians for the delta functions, we get

$$
\rho\left(\varepsilon_{F}\right)=\frac{a_{0}^{2} \gamma}{\pi^{2} v_{F} v_{2}} \sum_{n=1}^{2}\left[\ln \frac{\Lambda^{2}}{\left[\mu B \cos \left(\theta_{n}+\phi\right)\right]^{2}+\gamma^{2}}+2 \frac{\mu B \cos \left(\theta_{n}+\phi\right)}{\gamma} \tan ^{-1} \frac{\mu B \cos \left(\theta_{n}+\phi\right)}{\gamma}\right],
$$


where $\Lambda$ is a cutoff. The change in the DOS induced by the magnetic field is obtained by subtracting off the $B=0$ result,

$$
\Delta \rho\left(\varepsilon_{F}\right)=\frac{2 a_{0}^{2}}{\pi^{2} v_{F} v_{2}} \sum_{n=1}^{2}\left[\gamma \ln \frac{\gamma}{\sqrt{\left[\mu B \cos \left(\theta_{n}+\phi\right)\right]^{2}+\gamma^{2}}}+\mu B \cos \left(\theta_{n}+\phi\right) \tan ^{-1} \frac{\mu B \cos \left(\theta_{n}+\phi\right)}{\gamma}\right] .
$$

The change in the linear specific heat coefficient due to the magnetic field is then

$$
\Delta \gamma_{0}=\lim _{T \rightarrow 0} \frac{\Delta c_{v}}{T}=\frac{\pi^{2}}{3} k_{B}^{2} \Delta \rho\left(\epsilon_{F}\right) .
$$

[1] Y. Matsuda, K. Izawa, and I. Vekhter, Nodal structure of unconventional superconductors probed by angle resolved thermal transport measurements, J. Phys.: Condens. Matter 18, R705 (2006).

[2] I. Vekhter, P. J. Hirschfeld, J. P. Carbotte, and E. J. Nicol, Anisotropic thermodynamics of d-wave superconductors in the vortex state, Phys. Rev. B 59, 9023(R) (1999).

[3] A. B. Vorontsov and I. Vekhter, Unconventional superconductors under a rotating magnetic field. II. Thermal transport, Phys. Rev. B 75, 224502 (2007).

[4] T. Das, A. B. Vorontsov, I. Vekhter, and M. J. Graf, Field-angleresolved anisotropy in superconducting $\mathrm{CeCoIn}_{5}$ using realistic Fermi surfaces, Phys. Rev. B 87, 174514 (2013).

[5] K. Izawa, H. Yamaguchi, T. Sasaki, and Y. Matsuda, Superconducting Gap Structure of $\kappa$-(BEDT-TTF $)_{2} \mathrm{Cu}(\mathrm{NCS})_{2}$ Probed by Thermal Conductivity Tensor, Phys. Rev. Lett. 88, 027002 (2001).

[6] T. Watanabe, K. Izawa, Y. Kasahara, Y. Haga, Y. Onuki, P. Thalmeier, K. Maki, and Y. Matsuda, Superconducting gap function in antiferromagnetic heavy-fermion $\mathrm{UPd}_{2} \mathrm{Al}_{3}$ probed by angle-resolved magnetothermal transport measurements, Phys. Rev. B 70, 184502 (2004).

[7] D. Y. Kim, S.-Z. Lin, F. Weickert, E. D. Bauer, F. Ronning, J. D. Thompson, and R. Movshovich, Resonances in the FieldAngle-Resolved Thermal Conductivity of CeCoIn ${ }_{5}$, Phys. Rev. Lett. 118, 197001 (2017).

[8] F. Yu, M. B. Salamon, A. J. Leggett, W. C. Lee, and D. M. Ginsberg, Tensor Magnetothermal Resistance in $\mathrm{YBa}_{2} \mathrm{Cu}_{3} \mathrm{O}_{7-x}$ via Andreev Scattering of Quasiparticles, Phys. Rev. Lett. 74, 5136 (1995).

[9] H. Aubin, K. Behnia, M. Ribault, R. Gagnon, and L. Taillefer, Angular Position of Nodes in the Superconducting Gap of YBCO, Phys. Rev. Lett. 78, 2624 (1997).

[10] R. Ocaña and P. Esquinazi, Thermal conductivity tensor in $\mathrm{YBa}_{2} \mathrm{Cu}_{3} \mathrm{O}_{7-x}$ : Effects of a planar magnetic field, Phys. Rev. B 66, 064525 (2002).

[11] K. Izawa, H. Takahashi, H. Yamaguchi, Y. Matsuda, M. Suzuki, T. Sasaki, T. Fukase, Y. Yoshida, R. Settai, and Y. Onuki, Superconducting Gap Structure of Spin-Triplet Superconductor $\mathrm{Sr}_{2} \mathrm{RuO}_{4}$ Studied by Thermal Conductivity, Phys. Rev. Lett. 86, 2653 (2001).

[12] G. E. Volovik, Superconductivity with lines of GAP nodes: density of states in the vortex, JETP Lett. 58, 469 (1993).

[13] J. M. Riley, F. Mazzola, M. Dendzik, M. Michiardi, T. Takayama, L. Bawden, C. Granerød, M. Leandersson, T. Balasubramanian, M. Hoesch, T. K. Kim, H. Takagi, W. Meevasana, P. Hofmann, M. S. Bahramy, J. W. Wells, and
P. D. C. King, Direct observation of spin-polarized bulk bands in an inversion-symmetric semiconductor, Nat. Phys. 10, 835 (2014).

[14] D. Santos-Cottin, M. Casula, G. Lantz, Y. Klein, L. Petaccia, P. L. Fèvre, F. Bertran, E. Papalazarou, M. Marsi, and A. Gauzzi, Rashba coupling amplification by a staggered crystal field, Nat. Commun. 7, 11258 (2016).

[15] M. Gehlmann, I. Aguilera, G. Bihlmayer, E. Młyńczak, M. Eschbach, S. Döring, P. Gospodarič, S. Cramm, B. Kardynał, L. Plucinski, S. Blügel, and C. M. Schneider, Quasi 2D electronic states with high spin-polarization in centrosymmetric $\mathrm{MoS}_{2}$ bulk crystals, Sci. Rep. 6, 26197 (2016).

[16] E. Razzoli, T. Jaouen, M.-L. Mottas, B. Hildebrand, G. Monney, A. Pisoni, S. Muff, M. Fanciulli, N. C. Plumb, V. A. Rogalev, V. N. Strocov, J. Mesot, M. Shi, J. Dil, H. Beck, and P. Aebi, Selective Probing of Hidden Spin-Polarized States in InversionSymmetric Bulk $\mathrm{MoS}_{2}$, Phys. Rev. Lett. 118, 086402 (2017).

[17] S.-L. Wu, K. Sumida, K. Miyamoto, K. Taguchi, T. Yoshikawa, A. Kimura, Y. Ueda, M. Arita, M. Nagao, S. Watauchi, I. Tanaka, and T. Okuda, Direct evidence of hidden local spin polarization in a centrosymmetric superconductor $\mathrm{LaO}_{0.55} \mathrm{~F}_{0.45} \mathrm{BiS}_{2}$, Nat. Commun. 8, 1919 (2017).

[18] W. Yao, E. Wang, H. Huang, K. Deng, M. Yan, K. Zhang, K. Miyamoto, T. Okuda, L. Li, Y. Wang, H. Gao, C. Liu, W. Duan, and S. Zhou, Direct observation of spin-layer locking by local Rashba effect in monolayer semiconducting $\mathrm{PtSe}_{2}$ film, Nat. Commun. 8, 14216 (2017).

[19] X. Zhang, Q. Liu, J.-W. Luo, A. J. Freeman, and A. Zunger, Hidden spin polarization in inversion-symmetric bulk crystals, Nat. Phys. 10, 387 (2014).

[20] Q. Liu, X. Zhang, H. Jin, K. Lam, J. Im, A. J. Freeman, and A. Zunger, Search and design of nonmagnetic centrosymmetric layered crystals with large local spin polarization, Phys. Rev. B 91, 235204 (2015).

[21] L. Yuan, Q. Liu, X. Zhang, J.-W. Luo, S.-S. Li, and A. Zunger, Uncovering and tailoring hidden Rashba spinorbit splitting in centrosymmetric crystals, Nat. Commun. 10, 580 (2019).

[22] T. Das and A. V. Balatsky, Engineering three-dimensional topological insulators in Rashba-type spin-orbit coupled heterostructures, Nat. Commun. 4, 1972 (2013).

[23] X.-Y. Dong, J.-F. Wang, R.-X. Zhang, W.-H. Duan, B.-F. Zhu, J. O. Sofo, and C.-X. Liu, Electrically tunable multiple Dirac cones in thin films of the $(\mathrm{LaO})_{2}\left(\mathrm{SbSe}_{2}\right)_{2}$ family of materials, Nat. Commun. 6, 8517 (2015).

[24] M. Sigrist, D. F. Agterberg, M. H. Fischer, J. Goryo, F. Loder, S.-H. Rhim, D. Maruyama, Y. Yanase, T. Yoshida, 
and S. J. Youn, Superconductors with staggered noncentrosymmetricity, J. Phys. Soc. Jpn. 83, 061014 (2014).

[25] Y. Higashi, Y. Nagai, T. Yoshida, Y. Masaki, and Y. Yanase, Robust zero-energy bound states around a pair-density-wave vortex core in locally noncentrosymmetric superconductors, Phys. Rev. B 93, 104529 (2016).

[26] T. Yoshida, M. Sigrist, and Y. Yanase, Pair-density wave states through spin-orbit coupling in multilayer superconductors, Phys. Rev. B 86, 134514 (2012).

[27] C.-X. Liu, Unconventional Superconductivity in Bilayer Transition Metal Dichalcogenides, Phys. Rev. Lett. 118, 087001 (2017).

[28] T. Hitomi and Y. Yanase, Electric octupole order in bilayer ruthenate $\mathrm{Sr}_{3} \mathrm{Ru}_{2} \mathrm{O}_{7}$, J. Phys. Soc. Jpn. 83, 114704 (2014).

[29] T. Hitomi and Y. Yanase, Electric octupole order in bilayer Rashba system, J. Phys. Soc. Jpn. 85, 124702 (2016).

[30] K. Gotlieb, C.-Y. Lin, M. Serbyn, W. Zhang, C. L. Smallwood, C. Jozwiak, H. Eisaki, Z. Hussain, A. Vishwanath, and A. Lanzara, Revealing hidden spin-momentum locking in a hightemperature cuprate superconductor, Science 362, 1271 (2018).

[31] A. Audouard, C. Jaudet, D. Vignolles, R. Liang, D. A. Bonn, W. N. Hardy, L. Taillefer, and C. Proust, Multiple Quantum Oscillations in the de Haas-van Alphen Spectra of the Underdoped High-Temperature Superconductor $\mathrm{YBa}_{2} \mathrm{Cu}_{3} \mathrm{O}_{6.5}$, Phys. Rev. Lett. 103, 157003 (2009).

[32] S. E. Sebastian, N. Harrison, R. Liang, D. A. Bonn, W. N. Hardy, C. H. Mielke, and G. G. Lonzarich, Quantum Oscillations from Nodal Bilayer Magnetic Breakdown in the Underdoped High Temperature Superconductor $\mathrm{YBa}_{2} \mathrm{Cu}_{3} \mathrm{O}_{6+x}$, Phys. Rev. Lett. 108, 196403 (2012).

[33] N. Harrison, B. J. Ramshaw, and A. Shekhter, Nodal bilayersplitting controlled by spin-orbit interactions in underdoped high-Tc cuprates, Sci. Rep. 5, 10914 (2015).

[34] A. K. R. Briffa, E. Blackburn, S. M. Hayden, E. A. Yelland, M. W. Long, and E. M. Forgan, Fermi surface reconstruction and quantum oscillations in underdoped $\mathrm{YBa}_{2} \mathrm{Cu}_{3} \mathrm{O}_{7-x}$ modeled in a single bilayer with mirror symmetry broken by charge density waves, Phys. Rev. B 93, 094502 (2016).

[35] A. V. Maharaj, Y. Zhang, B. J. Ramshaw, and S. A. Kivelson, Quantum oscillations in a bilayer with broken mirror symmetry: A minimal model for $\mathrm{YBa}_{2} \mathrm{Cu}_{3} \mathrm{O}_{6+\delta}$, Phys. Rev. B 93, 094503 (2016).

[36] W. A. Atkinson, Microscopic model for the hidden Rashba effect in $\mathrm{YBa}_{2} \mathrm{Cu}_{3} \mathrm{O}_{6+x}$, Phys. Rev. B 101, 024513 (2020).

[37] V. Kaladzhyan, P. Simon, and C. Bena, Determining the spinorbit coupling via spin-polarized spectroscopy of magnetic impurities, Phys. Rev. B 94, 134511 (2016).

[38] Z. M. Raines, A. A. Allocca, and V. M. Galitski, Manifestations of spin-orbit coupling in a cuprate superconductor, Phys. Rev. B 100, 224512 (2019).

[39] K. Pasanai and W. A. Atkinson, Theory of (001) surface and bulk states in $\mathrm{Y}_{1-y} \mathrm{Ca}_{y} \mathrm{Ba}_{2} \mathrm{Cu}_{3} \mathrm{O}_{7-\delta}$, Phys. Rev. B 81, 134501 (2010).

[40] D. F. Agterberg, P. M. R. Brydon, and C. Timm, Bogoliubov Fermi Surfaces in Superconductors with Broken Time-Reversal Symmetry, Phys. Rev. Lett. 118, 127001 (2017).

[41] K. Yang and S. L. Sondhi, Response of a $d_{x^{2}-y^{2}}$ superconductor to a Zeeman magnetic field, Phys. Rev. B 57, 8566 (1998).

[42] M. Smidman, M. B. Salamon, H. Q. Yuan, and D. F. Agterberg, Superconductivity and spin-orbit coupling in non- centrosymmetric materials: a review, Rep. Prog. Phys. 80, 036501 (2017).

[43] K. A. Moler, D. L. Sisson, J. S. Urbach, M. R. Beasley, A. Kapitulnik, D. J. Baar, R. Liang, and W. N. Hardy, Specic heat of $\mathrm{YBa}_{2} \mathrm{Cu}_{3} \mathrm{O}_{7-\delta}$, Phys. Rev. B 55, 3954 (1997).

[44] I. M. Vishik, W. S. Lee, F. Schmitt, B. Moritz, T. Sasagawa, S. Uchida, K. Fujita, S. Ishida, C. Zhang, T. P. Devereaux, and Z. X. Shen, Doping-Dependent Nodal Fermi Velocity of the High-Temperature Superconductor $\mathrm{Bi}_{2} \mathrm{Sr}_{2} \mathrm{CaCu}_{2} \mathrm{O}_{8+\delta}$ Revealed Using High-Resolution Angle-Resolved Photoemission Spectroscopy, Phys. Rev. Lett. 104, 207002 (2010).

[45] D. Fournier, G. Levy, Y. Pennec, J. L. McChesney, A. Bostwick, E. Rotenberg, R. Liang, W. N. Hardy, D. A. Bonn, I. S. Elfimov, and A. Damascelli, Loss of nodal quasiparticle integrity in underdoped $\mathrm{YBa}_{2} \mathrm{Cu}_{3} \mathrm{O}_{6+x}$, Nat. Phys. 6, 905 (2010).

[46] T. Yoshida and Y. Yanase, Topological $D+p$-wave superconductivity in Rashba systems, Phys. Rev. B 93, 054504 (2016).

[47] A. Daido and Y. Yanase, Paramagnetically induced gapful topological superconductors, Phys. Rev. B 94, 054519 (2016).

[48] A. Daido and Y. Yanase, Majorana flat bands, chiral Majorana edge states, and unidirectional Majorana edge states in noncentrosymmetric superconductors, Phys. Rev. B 95, 134507 (2017).

[49] H. Sumiyoshi and S. Fujimoto, Quantum thermal Hall effect in a time-reversal-symmetry-broken topological superconductor in two dimensions: Approach from bulk calculations, J. Phys. Soc. Jpn. 82, 023602 (2013).

[50] V. Ambegaokar and A. Griffin, Theory of the thermal conductivity of superconducting alloys with paramagnetic impurities, Phys. Rev. 137, A1151 (1965).

[51] A. C. Durst and P. A. Lee, Impurity-induced quasiparticle transport and universal-limit Wiedemann-Franz violation in d-wave superconductors, Phys. Rev. B 62, 1270 (2000).

[52] T. Qin, Q. Niu, and J. Shi, Energy Magnetization and the Thermal Hall Effect, Phys. Rev. Lett. 107, 236601 (2011).

[53] M. Sutherland, D. G. Hawthorn, R. W. Hill, F. Ronning, S. Wakimoto, H. Zhang, C. Proust, E. Boaknin, C. Lupien, L. Taillefer, R. Liang, D. A. Bonn, W. N. Hardy, R. Gagnon, N. E. Hussey, T. Kimura, M. Nohara, and H. Takagi, Thermal conductivity across the phase diagram of cuprates: Low-energy quasiparticles and doping dependence of the superconducting gap, Phys. Rev. B 67, 174520 (2003).

[54] T. Yamasaki, K. Yamazaki, A. Ino, M. Arita, H. Namatame, M. Taniguchi, A. Fujimori, Z.-X. Shen, M. Ishikado, and S. Uchida, Unmasking the nodal quasiparticle dynamics in cuprate superconductors using low-energy photoemission, Phys. Rev. B 75, 140513(R) (2007).

[55] S. H. Pan, J. P. O’Neal, R. L. Badzey, C. Chamon, H. Ding, J. R. Engelbrecht, Z. Wang, H. Eisaki, S. Uchida, A. K. Gupta, K.-W. Ng, E. W. Hudson, K. M. Lang, and J. C. Davis, Microscopic electronic inhomogeneity in the high-Tc superconductor $\mathrm{Bi}_{2} \mathrm{Sr}_{2} \mathrm{CaCu}_{2} \mathrm{O}_{8+x}$, Nature 413, 282 (2001).

[56] Ø. Fischer, M. Kugler, I. Maggio-Aprile, C. Berthod, and C. Renner, Scanning tunneling spectroscopy of high-temperature superconductors, Rev. Mod. Phys. 79, 353 (2007).

[57] R. Harris, P. J. Turner, S. Kamal, A. R. Hosseini, P. Dosanjh, G. K. Mullins, J. S. Bobowski, C. P. Bidinosti, D. M. Broun, R. Liang, W. N. Hardy, and D. A. Bonn, Phenomenology of $\hat{a}$-axis 
and $\hat{b}$-axis charge dynamics from microwave spectroscopy of highly ordered $\mathrm{YBa}_{2} \mathrm{Cu}_{3} \mathrm{O}_{6.50}$ and $\mathrm{YBa}_{2} \mathrm{Cu}_{3} \mathrm{O}_{6.993}$, Phys. Rev. B 74, 104508 (2006).

[58] D. Bonn, S. Kamal, K. Zhang, R. Liang, and W. Hardy, The microwave surface impedance of $\mathrm{YBa}_{2} \mathrm{Cu}_{3} \mathrm{O}_{7-\delta}$, J. Phys. Chem. Solids 56, 1941 (1995).

[59] D. Dulić, S. J. Hak, D. van der Marel, W. N. Hardy, A. E. Koshelev, R. Liang, D. A. Bonn, and B. A. Willemsen, Effects of Vortex Pinning and Thermal Fluctuations on the Josephson Plasma Resonance in $\mathrm{Tl}_{2} \mathrm{Ba}_{2} \mathrm{CaCu}_{2} \mathrm{O}_{8}$ and $\mathrm{YBa}_{2} \mathrm{Cu}_{3} \mathrm{O}_{6.5}$, Phys. Rev. Lett. 86, 4660 (2001).
[60] L. Zhu, W. A. Atkinson, and P. J. Hirschfeld, Power spectrum of many impurities in a $d$-wave superconductor, Phys. Rev. B 69, 060503(R) (2004).

[61] N. R. Lee-Hone, J. S. Dodge, and D. M. Broun, Disorder and superfluid density in overdoped cuprate superconductors, Phys. Rev. B 96, 024501 (2017).

[62] N. R. Lee-Hone, V. Mishra, D. M. Broun, and P. J. Hirschfeld, Optical conductivity of overdoped cuprate superconductors: Application to $\mathrm{La}_{2-x} \mathrm{Sr}_{x} \mathrm{CuO}_{4}$, Phys. Rev. B 98, 054506 (2018).

[63] M. Sigrist, Introduction to unconventional superconductivity in non-centrosymmetric metals, AIP Conf. Proc. 1162, 55 (2009). 